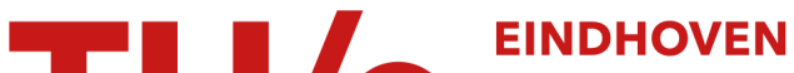 UNIVERSITY OF TECHNOLOGY
}

\section{Fluctuating lattice-Boltzmann model for complex fluids}

Citation for published version (APA):

Ollila, S. T. T., Denniston, C., Karttunen, M. E. J., \& Ala-Nissila, T. (2011). Fluctuating lattice-Boltzmann model for complex fluids. Journal of Chemical Physics, 134(6), 064902/1-19. https://doi.org/10.1063/1.3544360

DOI:

$10.1063 / 1.3544360$

Document status and date:

Published: 01/01/2011

\section{Document Version:}

Publisher's PDF, also known as Version of Record (includes final page, issue and volume numbers)

\section{Please check the document version of this publication:}

- A submitted manuscript is the version of the article upon submission and before peer-review. There can be important differences between the submitted version and the official published version of record. People interested in the research are advised to contact the author for the final version of the publication, or visit the $\mathrm{DOI}$ to the publisher's website.

- The final author version and the galley proof are versions of the publication after peer review.

- The final published version features the final layout of the paper including the volume, issue and page numbers.

Link to publication

\section{General rights}

Copyright and moral rights for the publications made accessible in the public portal are retained by the authors and/or other copyright owners and it is a condition of accessing publications that users recognise and abide by the legal requirements associated with these rights.

- Users may download and print one copy of any publication from the public portal for the purpose of private study or research.

- You may not further distribute the material or use it for any profit-making activity or commercial gain

- You may freely distribute the URL identifying the publication in the public portal.

If the publication is distributed under the terms of Article $25 f a$ of the Dutch Copyright Act, indicated by the "Taverne" license above, please follow below link for the End User Agreement:

www.tue.nl/taverne

Take down policy

If you believe that this document breaches copyright please contact us at:

openaccess@tue.nl

providing details and we will investigate your claim. 


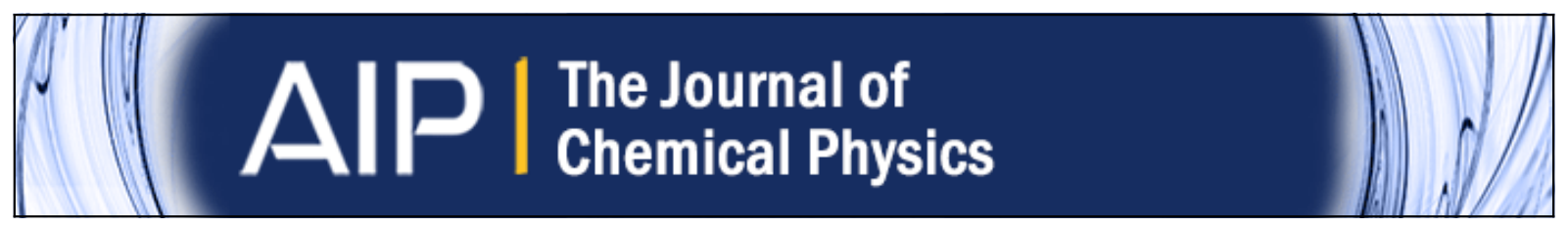

\section{Fluctuating lattice-Boltzmann model for complex fluids}

Santtu T. T. Ollila, Colin Denniston, Mikko Karttunen, and Tapio Ala-Nissila

Citation: The Journal of Chemical Physics 134, 064902 (2011); doi: 10.1063/1.3544360

View online: http://dx.doi.org/10.1063/1.3544360

View Table of Contents: http://scitation.aip.org/content/aip/journal/jcp/134/6?ver=pdfcov

Published by the AIP Publishing

\section{Articles you may be interested in}

Non-equilibrium work distributions from fluctuating lattice-Boltzmann model

AIP Conf. Proc. 1447, 195 (2012); 10.1063/1.4709947

Conformation and diffusion behavior of ring polymers in solution: A comparison between molecular dynamics, multiparticle collision dynamics, and lattice Boltzmann simulations

J. Chem. Phys. 135, 184901 (2011); 10.1063/1.3656761

Lattice-Boltzmann simulation of finite Reynolds number buoyancy-driven bubbly flows in periodic and wallbounded domains

Phys. Fluids 20, 103304 (2008); 10.1063/1.3001728

Velocity fluctuations and hydrodynamic diffusion in finite-Reynolds-number sedimenting suspensions

Phys. Fluids 20, 043305 (2008); 10.1063/1.2903623

Lattice-Boltzmann simulations of the dynamics of polymer solutions in periodic and confined geometries

J. Chem. Phys. 122, 094902 (2005); 10.1063/1.1854151

How can you REACH $100 \%$

of researchers at the Top 100

Physical Sciences Universities?

With The Journal of Chemical Physics.

AIP $\left.\right|_{\text {Chemical Physics }} ^{\text {The Journal of }}$

THERE'S POWER IN NUMBERS. Reach the world with AIP Publishing.

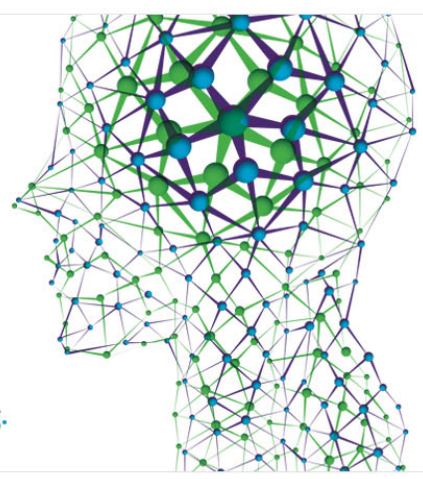




\title{
Fluctuating lattice-Boltzmann model for complex fluids
}

\author{
Santtu T. T. Ollila, ${ }^{1,2, a)}$ Colin Denniston, ${ }^{2, b)}$ Mikko Karttunen, ${ }^{2, c)}$ and Tapio Ala-Nissila ${ }^{1,3, d)}$ \\ ${ }^{1}$ Department of Applied Physics, Aalto University School of Science and Technology, P.O. Box 11000, \\ FIN-00076 Aalto, Espoo, Finland \\ ${ }^{2}$ Department of Applied Mathematics, The University of Western Ontario, London, Ontario, N6A 5B8, Canada \\ ${ }^{3}$ Department of Physics, Brown University, Providence, Rhode Island 02912-1843 USA
}

(Received 20 July 2010; accepted 31 December 2010; published online 10 February 2011)

\begin{abstract}
We develop and test numerically a lattice-Boltzmann (LB) model for nonideal fluids that incorporates thermal fluctuations. The fluid model is a momentum-conserving thermostat, for which we demonstrate how the temperature can be made equal at all length scales present in the system by having noise both locally in the stress tensor and by shaking the whole system in accord with the local temperature. The validity of the model is extended to a broad range of sound velocities. Our model features a consistent coupling scheme between the fluid and solid molecular dynamics objects, allowing us to use the LB fluid as a heat bath for solutes evolving in time without external Langevin noise added to the solute. This property expands the applicability of LB models to dense, strongly correlated systems with thermal fluctuations and potentially nonideal equations of state. Tests on the fluid itself and on static and dynamic properties of a coarse-grained polymer chain under strong hydrodynamic interactions are used to benchmark the model. The model produces results for singlechain diffusion that are in quantitative agreement with theory. () 2011 American Institute of Physics. [doi:10.1063/1.3544360]
\end{abstract}

\section{INTRODUCTION}

Submicron dynamics of suspensions are dictated by solvent-mediated long-range interactions and Brownian motion. The idea of the origin of Brownian motion being in the solvent surrounding the suspended particles dates back to Einstein's original work. ${ }^{1}$ Diffusion of lone colloids is often modeled using Brownian dynamics, but there are many collective phenomena in colloidal physics that require both hydrodynamic interactions and thermal fluctuations. ${ }^{2}$ Capturing both the rheology and thermal fluctuations in complex fluid simulations requires efficient computational algorithms.

Mesoscale continuum-solvent models, such as the latticeBoltzmann (LB) model, ${ }^{3,4}$ have become established tools for studies of complex fluids. ${ }^{5-11}$ However, they are mean-field models and typically contain no thermal fluctuations. In addition to their obvious importance to behavior near the critical point, there are many instances where thermal fluctuations play a role. ${ }^{12-14}$ The omission of thermal noise is also in sharp contrast to particle-based models, such as molecular dynamics (MD), dissipative particle dynamics (DPD), and stochastic rotation dynamics $(\mathrm{SRD})^{4}$ that contain fluctuations inherently. However, SRD and DPD are typically restricted $^{15,16}$ to a Schmidt number $S c$, the ratio of the diffusive momentum transfer rate to the diffusive mass transfer rate, well below 10 which is considerably lower than $S c \approx 460$ corresponding to water at S.T.P., ${ }^{17}$ although work on this aspect continues. ${ }^{18,19}$

\footnotetext{
a)Electronic mail: santtu.ollila@tkk.fi.

b)Electronic mail: cdennist@uwo.ca.

c) Electronic mail: mkarttu@uwo.ca.

d) Electronic mail: tapio.ala-nissila@tkk.fi.
}

Thermal fluctuations were first implemented in the LB model by $\operatorname{Ladd}^{20}$ in an effort to have solid particles undergo Brownian motion. Following Landau and Lifschitz, ${ }^{21}$ Ladd applied noise to the fluid stress tensor-an approach valid close to equilibrium. ${ }^{22}$ However, discretization of the Boltzmann transport equation ${ }^{23}$ to the LB equation creates lattice-specific independent degrees of freedom dubbed "ghosts" 24 that do not enter the macroscopic equations of mass and momentum conservation. As the moments of the LB equation corresponding to momentum and energy fluxes are coupled, noise applied only to the stress tensor leaks to higher-order ghost moments leading to poor temperature reproduction. This is likely because the isothermal LB model does not conserve energy. Adhikari et al. found that thermalization of ghosts improves local temperature reproduction in the isothermal LB model. ${ }^{25}$ Statistical mechanical foundation for the fluctuating LB model was provided by Dünweg et al. ${ }^{26}$ for LB models with equilibria accurate to second order in velocities under the assumption that the speed of sound $v_{s}$ in the model was $1 / \sqrt{3}$ in lattice units.

Fluctuating LB models have been applied to colloidal systems $^{25,27,28}$ using a bounce-back rule to account for the surface of the colloid, which has proven reasonable for particle radii $a>2.4 \Delta x$, where $\Delta x$ is the resolution of the LB mesh. ${ }^{28}$ Alternatively, in studies of rigid point ${ }^{29-31}$ and composite particles, ${ }^{32}$ a velocity coupling designed to make the particles track the fluctuating fluid velocity field was used and external Langevin noise was added to the particle phase. Ahlrichs and Dünweg simulated a polymer composed of point particles with external Langevin noise applied independently to all monomers. ${ }^{30}$ Their solvent was a fluctuating LB fluid as per Ref. 20 (without ghost noise) and they used a form of velocity coupling that resulted in an a priori chosen diffusion 
coefficient for a point particle. ${ }^{29,30}$ They observed correct long-time behavior and obtained good scaling results. ${ }^{30}$ More recently, Iwashita et al. ${ }^{33}$ applied Langevin noise to the polymer the same way as in Ref. 30, but not to the solvent, which in their case was a direct discretization of the incompressible nonfluctuating Navier-Stokes equation with a body force term coupling the two. Their results suggest that if one is only interested in correct behavior in the long-time limit (hydrodynamic tails), the only source of noise being in the particles' equation of motion suffices also for coarse-grained polymers. Most recently, Pham et al. ${ }^{31}$ used the same LB model with external Langevin noise. They found good agreement with Brownian dynamics simulations and scaling predictions only when they thermalized the ghost modes. In all these works, the speed of sound $v_{s}$ was set to $(\Delta x / \Delta t) / \sqrt{3}$, which simplifies the viscosity tensor.

Despite its apparent success, the coupling between the LB fluid and MD particles has subtleties arising from the nature of the coupling itself and whether one uses point or composite particles. In an accompanying paper, ${ }^{34}$ we establish criteria under which particles have a well-defined hydrodynamic radius, independent of means of measurement. Previous works do not fulfill all these criteria, which should not, however, be necessary for dilute systems. Yet, from a fundamental point of view, the existing schemes are unsatisfactory in that if the hydrodynamic coupling were physically correct, the LB fluid should suffice as the only thermostat to the particle phase.

The purpose of this paper is to establish how thermal fluctuations can be included in the LB method in a way that allows, first, one to use a broad range of values for the speed of sound even with fluctuations in the system and, second, the LB fluid to function as a heat bath even for a suspension of high solute concentration. The distinct difference to earlier approaches is: we do not add Langevin noise to any solute particles but, in keeping with Einstein's original idea, they pick up the correct temperature by basking in the fluid. This is highly relevant if one is to study systems where the solvent molecules are strongly correlated as their degrees of freedom cannot be assumed independent of one another.

In Sec. II, we present relevant aspects of the theory of thermal fluctuations in fluids. We build our isothermal model on previous approaches in Sec. III. The model is subjected to tests in Sec. IV, demonstrating how our scheme thermalizes fluctuations at all wavelengths. Moreover, we show that our fluid functions as a proper heat bath for solid inclusions and in particular exhibits quantitative agreement between theory and simulations for polymer diffusion and dynamic scaling.

\section{THEORY}

Thermal noise in a continuum description of a fluid is complicated because one must conserve mass and momentum globally yet still produce correct local fluctuations in the mass density $\rho$ and momentum density $\rho \mathbf{u}$. As a result, thermal fluctuations in fluids are much less studied and understood. Local fluctuations in mass and momentum arise through coupling to stress fluctuations in Navier-Stokes equations ${ }^{21}$ (summation over repeated indices is assumed),

$$
\partial_{t} \rho+\partial_{\beta}\left(\rho u_{\beta}\right)=0 ; \quad \partial_{t}\left(\rho u_{\alpha}\right)+\partial_{\beta}\left(\rho u_{\alpha} u_{\beta}\right)=\partial_{\beta} \sigma_{\alpha \beta},
$$

where the stress tensor $\sigma_{\alpha \beta}$ is given by

$$
\sigma_{\alpha \beta}=-P_{\alpha \beta}+\eta_{\alpha \beta \gamma \nu} \partial_{\gamma} u_{v}+s_{\alpha \beta} .
$$

In this case, the pressure tensor $P_{\alpha \beta}$, or nondissipative stress, can describe a nonideal fluid (e.g., $P_{o} \equiv \operatorname{Tr} P / 3$ could be the van der Waals equation of state) and can include gradient terms (potentially off-diagonal elements) capable of describing (diffuse) interfaces. ${ }^{5,6}$ The fluctuations in the stress enter through the fluctuating stress tensor $s_{\alpha \beta}$. In most cases the viscosity tensor $\eta_{\alpha \beta \gamma \nu}$ of the dissipative stress is fairly straightforward, containing only shear $\eta$ and bulk $\Lambda$ viscosity terms, ${ }^{35}$

$$
\eta_{\alpha \beta \gamma \nu}=\eta\left[\delta_{\alpha \gamma} \delta_{\beta \nu}+\delta_{\alpha \nu} \delta_{\beta \gamma}-\frac{2}{3} \delta_{\alpha \beta} \delta_{\gamma \nu}\right]+\Lambda \delta_{\alpha \beta} \delta_{\gamma \nu} .
$$

However, it may involve more independent viscosity coefficients in some complex fluids such as liquid crystals. The fluctuating stress $s_{\alpha \beta}$ is related to the viscosity through a fluctuation-dissipation relation, ${ }^{35}$

$$
\left\langle s_{\alpha \beta}(\mathbf{r}, t) s_{\gamma \nu}\left(\mathbf{r}^{\prime}, t^{\prime}\right)\right\rangle=2 \eta_{\alpha \beta \gamma \nu} k_{\mathrm{B}} T \delta\left(\mathbf{r}-\mathbf{r}^{\prime}\right) \delta\left(t-t^{\prime}\right) .
$$

Note that in general the diagonal components of $s_{\alpha \beta}$ will be correlated as will be discussed in Sec. III B.

As long as we reach local equilibrium, and have no velocity-dependent forces in our system, the equipartition theorem ${ }^{23}$ in a particle system tells us that fluctuations of the particle velocity $\delta \mathbf{v}$ obey $\left\langle\delta v_{\alpha} \delta v_{\beta}\right\rangle=\left(k_{B} T / M\right) \delta_{\alpha \beta}$, where $M$ is the mass of the particle. This is most often interpreted in a continuum system in the form

$$
\left\langle\delta u_{\alpha}\left(\mathbf{r}_{1}\right) \delta u_{\beta}\left(\mathbf{r}_{2}\right)\right\rangle=\frac{k_{\mathrm{B}} T}{\rho} \delta_{\alpha \beta} \delta\left(\mathbf{r}_{1}-\mathbf{r}_{2}\right),
$$

where $\delta u_{\alpha}=\left\langle\left(u_{\alpha}-\left\langle u_{\alpha}\right\rangle\right)\right\rangle$. Note, however, that Eq. (5) contains the additional assumption that the velocity fluctuations are delta function correlated, something not strictly required by the equipartition theorem and not generally true at microscopic distances. Ignoring these short distance correlations is justified when the distances involved are all much greater than the velocity-velocity correlation length and this is what we will assume here. Work is underway by other groups to relax this assumption and build velocity correlations into the thermal noise. ${ }^{36,37}$ Also, some caveats pertain to this formula in actual numerical simulations, in which the spatial Dirac delta function must be replaced by some finite volume $L_{s}^{3}$, where it approaches $1 / L_{s}^{3}$. In order to calculate the averages involved in $\delta u_{\alpha}$, one must calculate $u_{\alpha}$ and $\left\langle u_{\alpha}\right\rangle$ in a finite volume $L_{s}^{3}$, which on the discrete simulation mesh must be between the volume of a plaquette of the mesh $\Delta x^{3}$ and the system volume $L^{3}$, where $L$ is the linear size of the system. The fluid temperature $T\left(L_{s}\right)$ associated with a subvolume $L_{s}^{3}$ is thus determined from (here $\left\langle u_{\alpha}\right\rangle=0$ )

$$
\frac{1}{2} \sum_{\alpha}\left\langle\frac{\left[\sum_{\mathbf{x} \in L_{s}^{3}} \rho(\mathbf{x}) u_{\alpha}(\mathbf{x})\right]^{2}}{\sum_{\mathbf{x} \in L_{s}^{3}} \rho(\mathbf{x})}\right\rangle=\frac{3}{2} k_{\mathrm{B}} T\left(L_{s}\right) .
$$

Navier-Stokes relations conserve the total momentum of the system so that $\delta u_{\alpha}$ is zero if the discrete volume is that of the 
entire system whereas in the strictest continuum interpretation this should only be true for an infinite system. At the other end of the spectrum, at the highest wavevector supported by the lattice, $k=2 \pi / \Delta x$, one would hope Eq. (5) to be at least approximately valid as long as the mesh spacing were sufficiently larger than the microscopic velocity-velocity correlation length of the fluid. Thus, conservation of momentum results in the $T\left(L_{s}\right)$ defined in Eq. (6) not to be the true local temperature. We will examine this point more closely in Sec. IV A.

The fluctuation-dissipation relation of Eq. (4) is frequently used in MD to measure the viscosity based on fluctuations in the stress but is seldom used in modeling the fluctuating Navier-Stokes equation itself. The reason for this omission is the difficulty in solving this complex set of equations. Navier-Stokes equations are already very complicated and computational fluid dynamics calculations can easily strain the resources of the largest supercomputers. Next, we introduce the LB method used to solve these equations.

\section{FLUID MODEL}

\section{A. Lattice-Boltzmann model}

The LB algorithms are based on solving an approximation of the Boltzmann transport equation on a structured lattice with sites $\mathbf{x}=(i, j, k) \Delta x$. In the simplest single-relaxation time approximation, these algorithms simulate the Lattice Bhatnagar-Gross-Krook (LBGK) equation, ${ }^{38}$ typically of the form

$$
D_{i} f_{i} \equiv\left(\partial_{t}+e_{i \alpha} \partial_{\alpha}\right) f_{i}=-\frac{1}{\tau}\left(f_{i}-f_{i}^{\mathrm{eq}}\right)+W_{i}
$$

where $\mathbf{e}_{i}$ are the discrete velocities that transport material to neighboring lattice sites in the discrete time $\Delta t$ and their dimension is the lattice velocity $v_{c} \equiv \Delta x / \Delta t, D_{i}$ is the material derivative in direction $\mathbf{e}_{i}, \tau$ is the relaxation time, $f_{i}(\mathbf{x}, t)$ is the discretized distribution function representing the partial density of material moving with velocity $\mathbf{e}_{i}=\left(e_{i x}, e_{i y}, e_{i z}\right)$, and $f_{i}^{\mathrm{eq}}(\mathbf{x}, t)$ is the equilibrium value of $f_{i}$. In fact, $f_{i}^{\text {eq }}$ is typically a suitably weighted expansion of the MaxwellBoltzmann (MB) distribution in the discrete velocities, ${ }^{39}$ but alternatives have also been developed. ${ }^{40}$ We have included a general forcing term $W_{i}(\mathbf{x}, t)$ in Eq. (7) as per Ref. 34. We defer presenting a finite-difference form of Eq. (7) until later as our noise implementation of Sec. III B is based on the continuous BGK equation. ${ }^{38}$ These LBGK equations are much simpler to simulate than the Navier-Stokes equations (they can be cast as a system of ordinary differential equations in the Lagrangian picture) at the cost of having to simulate more equations. That is, one typically needs $n=15$ partial densities $f_{i}$ in three dimensions instead of the three spatial components of momentum and one scalar density of the Navier-Stokes equations. The degree to which the higher-order moments in velocities are reproduced correctly depends on the underlying lattice structure. The more vectors $\mathbf{e}_{i}$ there are, the more isotropic the lattice becomes and the higher moments of $f_{i}^{\text {eq }}$ can be made to correspond to those of the MB distribution.
The shorthand notation $\mathrm{D} d \mathrm{Q} n$ tells the number of spatial dimensions $d$ in the lattice and the number of lattice vectors $n$.

How Eq. (7) is related to Eq. (1) or its approximative form becomes evident when one considers moments of the discretized distribution function and expands Eq. (7) in space and time using the Chapman-Enskog expansion, see, e.g., Ref. 3. The mass and momentum densities are defined by

$$
\begin{aligned}
\rho(\mathbf{x}, t) & =M^{0}(\mathbf{x}, t)=\sum_{i} f_{i}(\mathbf{x}, t), \\
\rho(\mathbf{x}, t) u_{\alpha}(\mathbf{x}, t) & =M^{a}(\mathbf{x}, t)=\sum_{i} e_{i \alpha} f_{i}(\mathbf{x}, t),
\end{aligned}
$$

where $x(a=1), y(a=2)$, and $z(a=3)$ components of momentum define $M^{1}-M^{3}$. These linear functions of the $f_{i}$ can be generalized to give higher-order moments $M^{a}(\mathbf{x}, t)$ as

$$
M^{a}(\mathbf{x}, t)=\sum_{i} m_{i}^{a} f_{i}(\mathbf{x}, t)
$$

where $m_{i}^{a}$, given in Appendix A for the D3Q15 model, corresponds to moments of the velocity vectors $\mathbf{e}_{i}$ so that Eq. (9) provides the discrete versions of integrals such as $\int\left(v_{\alpha} v_{\beta} \ldots\right) f(\mathbf{r}, \mathbf{v}, t) \mathrm{d} \mathbf{v}$ in continuum transport theory. Equation (9) is invertible to give the $f_{i}$ in terms of the densities as

$$
f_{i}(\mathbf{x}, t)=w_{i} \sum_{a} m_{i}^{a} M^{a}(\mathbf{x}, t) N^{a},
$$

where the $w_{i}$ are weight factors and the $N^{a}$ are normalization constants given in Appendix A. Equation (10) is general meaning that any function may be expanded in terms of corresponding physical moments and we use this to define the equilibrium distribution,

$$
f_{i}^{\mathrm{eq}}(\mathbf{x}, t)=w_{i} \sum_{a} m_{i}^{a} M_{\mathrm{eq}}^{a}(\mathbf{x}, t) N^{a},
$$

where the moments $M_{\mathrm{eq}}^{a}$ for the D3Q15 model are given in Table I. We will use a similar expression later for the fluctuations $\bar{\xi}_{i}(\mathbf{x}, t)$ in $f_{i}^{\mathrm{eq}}(\mathbf{x}, t)$. Mass and momentum conservations are imposed by requiring that the first four moments $\left(M^{0}\right.$ $-M^{3}$ ) of the equilibrium distribution are equal to those of $f_{i}$. By construction, this makes the right-hand side of Eq. (7) zero as one calculates its zeroth or any of the firstorder velocity moments. We have included the necessary numerical values for implementing our model on the D3Q15 lattice ${ }^{41}$ structure in Appendix A together with pseudocode in Appendix B. Here we present the theory in general form.

Plugging Eq. (10) into Eq. (7) and making note of the fact that (cf. Appendix A and Table II therein)

$$
N^{a} \sum_{i} w_{i} m_{i}^{a} m_{i}^{b}=\delta_{a b},
$$

gives the local density evolution equations for the $n$ different $M^{a}(\mathbf{x}, t)$,

$$
\begin{aligned}
\partial_{t} M^{a}+\partial_{\alpha}\left(\sum_{i} m_{i}^{a} e_{i \alpha} f_{i}\right)= & -\frac{1}{\tau}\left(M^{a}-M_{\mathrm{eq}}^{a}\right) \\
& +\sum_{i} m_{i}^{a} W_{i},
\end{aligned}
$$


TABLE I. Moments of the equilibrium distribution $M_{\mathrm{eq}}^{a}$ in the D3Q15 model and of the continuum noise distribution $\bar{\zeta}^{a}$.

\begin{tabular}{cccccccccccccc}
\hline \hline$a$ & 0 & 1 & 2 & 3 & 4 & 5 & 6 & 7 & 8 & 9 & $10-12$ & 13 \\
\hline$M_{\mathrm{eq}}^{a}$ & $\rho$ & $\rho u_{x}$ & $\rho u_{y}$ & $\rho u_{z}$ & $P_{x x}+$ & $P_{y y}+$ & $P_{z z}+$ & $P_{x y}+\rho u_{x} u_{y}$ & $P_{y z}+\rho u_{y} u_{z}$ & $P_{x z}+\rho u_{x} u_{z}$ & 0 & $\rho u_{x} u_{y} u_{z}$ & $K^{\mathrm{eq}}$ \\
$\bar{\zeta}^{a}$ & 0 & 0 & 0 & 0 & $s_{x x}$ & $s_{y y}^{2}$ & $s_{z z}^{2}$ & $s_{x y}$ & $s_{y z}$ & $s_{x z}$ & $\bar{\zeta}^{a}$ & $\bar{\zeta}^{13}$ & $\bar{\zeta}^{14}$ \\
\hline \hline
\end{tabular}

where $M_{\mathrm{eq}}^{a}=\sum_{i} m_{i}^{a} f_{i}^{\mathrm{eq}}$. The forcing terms,

$$
W_{i}=p_{i}+\frac{1}{\tau} \bar{\xi}_{i}
$$

are split into two parts, of which the $p_{i}$ model external forces and the $\bar{\xi}_{i}$ thermal fluctuations internal to the fluid. The factor $1 / \tau$ is included in the definition of the $\bar{\xi}_{i}$ to make them consistent with the definitions of the equilibrium distribution in Eq. (7). The three lowest moments of the external forcing terms are chosen to be $\mathrm{be}^{34,42}$

$$
\begin{aligned}
& \sum_{i} p_{i}=0 ; \quad \sum p_{i} e_{i \alpha}=F_{\alpha} \\
& \sum_{i} p_{i} e_{i \alpha} e_{i \beta}=u_{\alpha} F_{\beta}+F_{\alpha} u_{\beta},
\end{aligned}
$$

where $F_{\alpha}$ is a force density external to the fluid modeled by the LB equations, which may contain contributions from gravity and/or a particle phase. The second moment is consistent with Boltzmann's equation (see Appendix in Ref. 34).

If we now insist that $f_{i}^{\text {eq }}$ is constructed so that $M_{\mathrm{eq}}^{0}$ $=\rho, M_{\mathrm{eq}}^{a}=\rho u_{\alpha}$ for $a \in\{1,2,3\}$ and $M_{\mathrm{eq}}^{a}=P_{\alpha \beta}+\rho u_{\alpha} u_{\beta}$ for $a \in\{4,5, \ldots, 9\}$, then by performing the ChapmanEnskog expansion for Eq. (7), the equations of motion for the zeroth and first-order moments become in the hydrodynamic limit, ${ }^{3}$

$$
\begin{aligned}
& \partial_{t} \rho+\partial_{\beta}\left(\rho u_{\beta}\right)=0+\mathcal{O}\left(\partial^{3}\right) ; \\
& \partial_{t}\left(\rho u_{\alpha}\right)+\partial_{\beta} \Pi_{\alpha \beta}=0+\mathcal{O}\left(\partial^{2}\right),
\end{aligned}
$$

where $\Pi_{\alpha \beta}=\sum_{i} f_{i} e_{i \alpha} e_{i \beta}$ is the second moment. The explicit expression for the Navier-Stokes equation of Eq. (16b) reads $^{3,6}$

$$
\begin{aligned}
\partial_{t}\left(\rho u_{\alpha}\right)+\partial_{\beta}\left(\rho u_{\alpha} u_{\beta}\right)= & \partial_{\alpha}\left(-P_{\alpha \beta}+s_{\alpha \beta}\right)+F_{\alpha} \\
& +\partial_{\beta}\left(\eta\left(\partial_{\alpha} u_{\beta}+\partial_{\beta} u_{\alpha}-\frac{2}{3} \partial_{\gamma} u_{\gamma} \delta_{\alpha \beta}\right)\right. \\
& \left.+\Lambda \partial_{\gamma} u_{\gamma} \delta_{\alpha \beta}\right),
\end{aligned}
$$

where for the ideal gas equation of state the pressure tensor $P_{\alpha \beta}=P_{o} \delta_{\alpha \beta}=\rho v_{s}^{2} \delta_{\alpha \beta}$ is diagonal, and $v_{s}$ is the speed of sound. The shear viscosity is then $\eta=\rho \tau v_{c}^{2} / 3$ and the bulk viscosity $\Lambda=\eta\left(5 / 3-\left(3 / v_{c}^{2}\right) \partial P_{o} / \partial \rho\right)=\eta(5 / 3$ $-3 v_{s}^{2} / v_{c}^{2}$ ). The fluctuating stress $s_{\alpha \beta}$ enters Eq. (17) through the second moment of $\bar{\xi}_{i}$ as described in Sec. III B.

Before we discuss thermal fluctuations in the model, we must discuss their local dynamics and feedback structure. An analysis included in Appendix A of the dynamics of highorder densities $M^{a}, a>4$, for the D3Q15 model leads to the following relation between the leading-order stresses, $\Pi_{\alpha \beta}^{(0)}$ $=P_{\alpha \beta}+\rho u_{\alpha} u_{\beta}$, and leading-order ghost momentum densities written in lattice units $\left(v_{c}=1\right)$ :

$$
\begin{aligned}
& \frac{2}{3} \nabla \cdot \cdot\left[\begin{array}{ccc}
\rho\left(u_{y}^{2}+u_{z}^{2}\right) / 2 & P_{x y}+\rho u_{x} u_{y} & P_{x z}+\rho u_{x} u_{z} \\
P_{x y}+\rho u_{x} u_{y} & \rho\left(u_{x}^{2}+u_{z}^{2}\right) / 2 & P_{x y}+\rho u_{y} u_{z} \\
P_{x z}+\rho u_{x} u_{z} & P_{y z}+\rho u_{y} u_{z} & \rho\left(u_{x}^{2}+u_{y}^{2}\right) / 2
\end{array}\right] \\
&+\frac{2}{9} \nabla\left(\operatorname{Tr} P-\rho+\frac{K^{\mathrm{eq}}}{\sqrt{2}}\right)=-\frac{1}{\tau} \mathbf{J}^{(1)},
\end{aligned}
$$

where $K^{\text {eq }}$ is the 14 th density at equilibrium, $M_{\text {eq }}^{14}$, and $\mathbf{J}^{(1)}$ is the nonequilibrium part of $\left(M^{12}, M^{10}, M^{11}\right)$ whose equilibrium value is zero. ${ }^{39}$ The value of $K^{\text {eq }}$ is not determined by any condition at this point. In the presence of fluctuating stresses $s_{\alpha \beta}$, however, this relation clearly demonstrates that the fluctuating stresses [which will appear in conjunction with the corresponding $P_{\alpha \beta}$ in Eq. (18)] will couple to $K^{\text {eq }}$-hence its value will be relevant.

Equation (18) is the equivalent to an expression in $2 \mathrm{D}$ derived by Dellar in Ref. 39. In the deterministic case that he studied, Dellar showed that the damping of stress fluctuations is related to the value of $K^{\text {eq }}$. Dellar set a nonzero value of $K^{\text {eq }}$ to remove high-frequency oscillations from the model in order to make his finite-difference scheme more "dissipative," and hence more stable. We do not do that here as the removal affects the fluctuations and thereby the temperature. Instead, we reserve the freedom to pick the amplitude $K_{0}$ of the 14th equilibrium moment,

$$
K^{\mathrm{eq}}=K_{0}(\rho-\operatorname{Tr}(P-s)),
$$

as we will be able to use it below to tune temperature reproduction in the model. This form is chosen since, if $\operatorname{Tr} P=\rho$ in lattice units, the damping effect described by Eq. (18) disappears for $K^{\text {eq }}=0$ without noise. ${ }^{39}$ Having determined the form of the densities $M_{\mathrm{eq}}^{a}$, we are at a position to construct the equilibria $f_{i}^{\text {eq }}$ using Eq. (10) and tune $K_{0}$. Next, we introduce fluctuations in the equilibrium populations.

\section{B. Thermal noise in the lattice-Boltzmann model}

Generally, LB works best when the fluid is essentially incompressible, ${ }^{24}$ but corrections can be added for fluids of nonuniform density. ${ }^{43}$ However, with thermal fluctuations present, density fluctuates locally ( $\delta \rho \neq 0$, but still small) and, therefore, the bulk viscosity coefficient is relevant. Previous LB studies ${ }^{20,25,26,29-31}$ incorporating noise have assumed the speed of sound squared $v_{s}^{2}=v_{c}^{2} / 3$, a choice which zeroes the viscosity coefficient of the $\partial_{\gamma} u_{\gamma}$ term in Eq. (17). We will illustrate the complications that arise when one 
uses a nontrivial bulk viscosity in the single relaxation time scheme (i.e., when $v_{s}^{2} \neq v_{c}^{2} / 3$ ), which allows straightforward generalization to the multirelaxation time D3Q15 and D3Q19 models $^{44}$ that permit arbitrary bulk viscosity.

As in Ref. 25, we implement our noise in Eq. (7) via the forcing terms $W_{i}$ of Eq. (14) which adds noise to the stress and ghost modes. The $\bar{\xi}_{i}$ are decomposed into physically meaningful continuum fluctuations in local densities and ghost densities using Eq. (10),

$$
\bar{\xi}_{i}(\mathbf{x}, t)=w_{i} \sum_{a} m_{i}^{a} \overline{\zeta^{a}}(\mathbf{x}, t) N^{a}
$$

The $\bar{\zeta}^{a}$ represent the local thermal fluctuations in the corresponding continuum physical and ghost densities $M_{\text {eq }}^{a}$ and are given in Table I. The first four moments, $\bar{\zeta}^{0}$ through $\bar{\zeta}^{3}$, must be identically zero [see Eq. (9) and (13)] as mass and momentum must be conserved exactly. The continuum noise terms $\bar{\zeta}^{4}$ through $\bar{\zeta}^{9}$ are the fluctuating stress terms $s_{\alpha \beta}$ of Eq. (16b). We emphasize that these continuum-limit fluctuations are not in general the lattice-level discrete-time stresses, but only related to them. We will show below that the latticelevel discrete-time stress fluctuations $\zeta^{a}$ depend, first, on the kinetic equation modeled [here Eq. (7)], second, the underlying lattice model [here D3Q15] and, third, the finitedifference scheme used [here Eq. (27)]. We will explain these dependencies in detail in the following passages. The remaining noises, $\zeta^{10}$ through $\zeta^{14}$, are related to the lattice-specific ghost modes that are independent degrees of freedom in the $w_{i}$-weighted basis defined by the 15 vectors $m^{a}$ subject to the orthogonality relation of Eq. (12).

Our starting point is that we want to reproduce the noise given in Eq. (4) locally at the lattice level leading to the reproduction of Eq. (5) also at the lattice level. The nontriviality in doing this arises from the three aforementioned dependencies. After the following three steps, we will have obtained expressions for the variances of each lattice-level discrete-time random variable $\zeta^{a}$.

Step 1. Identification as an Ornstein-Uhlenbeck process. In the theory of stochastic differential equations (SDEs), an equation of the form,

$$
\mathrm{d} X_{t}=\left(a(t)+b(t) X_{t}\right) \mathrm{d} t+\left(\alpha(t)+\beta(t) X_{t}\right) \mathrm{d} B_{t},
$$

is known as an Ornstein-Uhlenbeck (or Langevin) process for a stochastic variable $X_{t} \cdot{ }^{45}$ The functions $a(t), b(t), \alpha(t)$, and $\beta(t)$ are continuous processes adapted to the filtration of $X_{t}$, $B_{t}$ is a Brownian motion, and $\mathrm{d} B_{t}$ is the Wiener measure. The general solution to this equation is ${ }^{46}$

$$
\begin{aligned}
& X_{t^{\prime}}=U_{t^{\prime}}\left(X_{t}+\int_{t}^{t^{\prime}} \frac{a(s)-\beta(s) \alpha(s)}{U_{s}} \mathrm{~d} s+\int_{t}^{t^{\prime}} \frac{\alpha(s)}{U_{s}} \mathrm{~d} B_{s}\right) ; \\
& U_{t^{\prime}}=\exp \left(\int_{t}^{t^{\prime}} \beta(s) \mathrm{d} B_{s}+\int_{t}^{t^{\prime}}\left(b(s)-\frac{1}{2} \beta^{2}(s)\right) \mathrm{d} s\right) .
\end{aligned}
$$

We may take direct advantage of this result by identifying the $f_{i}$ in Eq. (7) as the process $X_{t}, a(t)$ as $(1 / \tau)\left(f_{i}^{\text {eq }}+\tau p_{i}\right), b(t)$ as $-1 / \tau, \alpha(t) \mathrm{d} B_{t}$ as $\left(\xi_{i} / \tau\right) \mathrm{d} t$ and $\beta(t)$ as identically zero to rewrite the solution of Eq. (7) at $t^{\prime}=t+\Delta t$ in the comoving frame $^{47}$ as

$$
\begin{aligned}
f_{i}(\mathbf{x} & \left.+\mathbf{e}_{i} \Delta t, t+\Delta t\right) \\
= & e^{-\Delta t / \tau} f_{i}(\mathbf{x}, t)+e^{-\Delta t / \tau} \int_{t}^{t+\Delta t} \frac{1}{\tau} e^{(s-t) / \tau} \\
& \quad \times g_{i}^{\mathrm{eq}}\left(\mathbf{x}+\mathbf{e}_{i} s, t+s\right) \mathrm{d} s+e^{-\Delta t / \tau} \int_{t}^{t+\Delta t} \frac{1}{\tau} e^{(s-t) / \tau} \bar{\xi}_{i} \mathrm{~d} t,
\end{aligned}
$$

where

$$
g_{i}^{\mathrm{eq}}=f_{i}^{\mathrm{eq}}+\tau p_{i},
$$

and we have made use of the fact that $U_{s}$ simplifies to $U_{s}$ $=\exp \left(\int_{t}^{s}-1 / \tau \mathrm{d} s\right)=\exp ((t-s) / \tau)$.

From Eq. (23), the variance in the noise term (the last term) integrated over one time step $\Delta t$ can be calculated to obtain

$$
\begin{aligned}
\operatorname{Var} & {\left[e^{-\Delta t / \tau} \int_{t}^{t+\Delta t} \frac{1}{\tau} e^{(s-t) / \tau} \bar{\xi}_{i} \mathrm{~d} t\right] } \\
& =\left\langle\bar{\xi}_{i}{ }^{2}\right\rangle \frac{(1-\exp (-2 \Delta t / \tau))}{2 \tau} \\
& \equiv\left\langle\bar{\xi}_{i}{ }^{2}\right\rangle q_{\mathrm{OU}}^{2},
\end{aligned}
$$

where we have assumed the continuum stochastic process $\bar{\xi}_{i}$ to have zero mean and that its standard deviation is approximately constant during the time step. This is the variance inherent to this SDE and $q_{\mathrm{OU}}^{2}$ defined in the last line of Eq. (25) is a factor that we must retain in the discrete-time and space random variables $\xi_{i}$ to ensure that fluctuations of the desired variance are present in the discrete version of the LBGK equation, Eq. (7), at every time step. As Eq. (20) relates $\bar{\xi}_{i}$ and $\bar{\zeta}^{a}$ linearly, the same factor relates $\zeta^{a}$ to $\bar{\zeta}^{a}$, i.e.,

$$
\left\langle\left(\zeta^{a}\right)^{2}\right\rangle \propto\left\langle\left(\overline{\zeta^{a}}\right)^{2}\right\rangle q_{\mathrm{OU}}^{2}
$$

Step 2. Identifying the contribution from the finitedifference scheme. The finite-difference scheme, we employ, is similar to one for the deterministic terms that can be found in Ref. 34. It is obtained by replacing $g_{i}^{\mathrm{eq}}\left(\mathbf{x}+\mathbf{e}_{i} s, t+s\right)$ in Eq. (23) by a Taylor series about $s=0$ and then directly integrating. The resulting time-evolution equation for the $f_{i}$ is

$$
\begin{aligned}
f_{i}(\mathbf{x} & \left.+\mathbf{e}_{i} \Delta t, t+\Delta t\right) \\
= & e^{-\Delta t / \tau} f_{i}(\mathbf{x}, t)+\left(1-e^{-\Delta t / \tau}\right) h_{i}^{\mathrm{eq}}(\mathbf{x}, t) \\
& +\Delta t\left(1-\frac{\tau}{\Delta t}\left(1-e^{-\Delta t / \tau}\right)\right) D_{t} h_{i}^{\mathrm{eq}}(\mathbf{x}, t) \\
& +\Delta t^{2}\left(\frac{\tau^{2}}{\Delta t^{2}}\left(1-e^{-\Delta t / \tau}\right)-\frac{\tau}{\Delta t}+\frac{1}{2}\right) D_{t}^{2} h_{i}^{\mathrm{eq}}(\mathbf{x}, t) \\
& +\mathcal{O}\left(\Delta t^{4}\right),
\end{aligned}
$$

where for computational efficiency and to save memory we have included the noise terms in the local equilibria which we now generalize as

$$
h_{i}^{\mathrm{eq}}=g_{i}^{\mathrm{eq}}+\xi_{i},
$$

where $\xi_{i}$ is the discrete noise process. We emphasize here that the discrete process $\xi_{i}$ is not the same as the continuum 
process $\bar{\xi}_{i}$ of Eqs. (7) and (20). The variance of $\xi_{i}$ is related to that of the continuum stochastic process $\bar{\xi}_{i}$ by $\left\langle\xi_{i}^{2}\right\rangle=$ $\left\langle\xi_{i}^{2}\right\rangle q_{\mathrm{OU}}^{2} / q_{\mathrm{FD}}^{2}$, where $q_{\mathrm{OU}}^{2}$ comes from Eq. (25) and the factor,

$$
q_{\mathrm{FD}}=\left(1-e^{-\Delta t / \tau}\right),
$$

is needed to cancel out the same factor on the second line of Eq. (27). This neglects the second-order terms that arise from Ito's lemma which we expect to be small. Later in Results, we will demonstrate that identifying $q_{\mathrm{FD}}$ as the only correction arising from the finite-difference algorithm is enough to capture the temperature dependence sufficiently accurately. We refer to Eq. (27) with only the first-order derivative term included as the first-order scheme and with both derivative terms as the second-order scheme. The more standard finitedifference scheme is obtained from Eq. (27) in the limit of small $\Delta t / \tau$ with the derivative terms neglected,

$$
\begin{aligned}
f_{i}\left(\mathbf{x}+\mathbf{e}_{i} \Delta t, t+\Delta t\right)= & f_{i}(\mathbf{x}, t) \\
& -\frac{\Delta t}{\tau}\left(f_{i}(\mathbf{x}, t)-h_{i}^{\mathrm{eq}}(\mathbf{x}, t)\right) .
\end{aligned}
$$

In practice, the standard scheme is equivalent to a secondorder accurate form using the trapezoidal rule, which formally requires one to define so-called auxiliary populations. ${ }^{48}$ The following analysis applies also to the standard scheme if $\tau$ in the viscosity, given below Eq. (17) is replaced by $\tau-\Delta t / 2$.

Step 3. Noise amplitudes in densities. What is left is to determine the noise correlation matrix with elements $\left\langle\zeta^{a}(\mathbf{x}, t) \zeta^{b}\left(\mathbf{x}^{\prime}, t^{\prime}\right)\right\rangle$ of the Gaussian random numbers with zero mean according to which the noise is added at the local lattice level. Analogous to Eq. (20) for the continuum-level fluctuations, the discrete-time lattice-level fluctuations obey

$$
\xi_{i}(\mathbf{x}, t)=w_{i} \sum_{a} m_{i}^{a} \zeta^{a}(\mathbf{x}, t) N^{a} .
$$

We may now write down the lattice-level version of Eq. (4) for our ordering of basis vectors $m^{a}$ (see Appendix A) by making use of the corrections from previous steps. We will come back to the general case below, but first consider the case where $v_{s}^{2}=v_{c}^{2} / 3$, which results in the fluctuating components of the stress tensor being independent random variables, and

$$
\begin{aligned}
\left\langle\left(\zeta^{a}\right)^{2}\right\rangle & =\left\langle\left(\zeta^{a}\right)^{2}\right\rangle \frac{q_{\mathrm{OU}}^{2}}{q_{\mathrm{FD}}^{2}}=\left\langle s_{\alpha \beta}^{2}\right) \frac{q_{\mathrm{OU}}^{2}}{q_{\mathrm{FD}}^{2}} \\
& =2 \eta k_{\mathrm{B}} T \frac{1}{\Delta x^{3} \Delta t} \frac{q_{\mathrm{OU}}^{2}}{q_{\mathrm{FD}}^{2}}, \quad a=7,8,9 ; \\
\left\langle\left(\zeta^{a}\right)^{2}\right\rangle & =\left\langle s_{\alpha \alpha}^{2}\right) \frac{q_{\mathrm{OU}}^{2}}{q_{\mathrm{FD}}^{2}}=4 \eta k_{\mathrm{B}} T \frac{1}{\Delta x^{3} \Delta t} \frac{q_{\mathrm{OU}}^{2}}{q_{\mathrm{FD}}^{2}}, \quad a=4,5,6,
\end{aligned}
$$

where the $1 /\left(\Delta x^{3} \Delta t\right)$ terms are the discrete spatial and temporal delta functions from Eq. (4). This formulation can be extended straightforwardly to more complicated collision matrices than $(-1 / \tau) \delta_{i j}$ of the BGK approximation. We would like to point out the necessity of subtracting $K_{0}$ times the trace of the noise, $K_{0}\left(\zeta^{4}+\zeta^{5}+\zeta^{6}\right)$, from $M_{\mathrm{eq}}^{14}=K^{\mathrm{eq}}$ as $K^{\text {eq }} \propto-K_{0} \operatorname{Tr} P$ in constructing the equilibria as in Eq. (10).

Both relations in Eq. (32) are instances of a general formula,

$$
\left\langle\left(\zeta^{a}\right)^{2}\right\rangle=\frac{18}{N^{a}} A_{\eta} \equiv \frac{18}{N^{a}} \eta k_{\mathrm{B}} T \frac{1}{\Delta x^{3}} \frac{1}{\Delta t} \frac{q_{\mathrm{OU}}^{2}}{q_{\mathrm{FD}}^{2}},
$$

which reduces to Eq. (32) when the corresponding normalization constants $N^{a}$ given in Eq. (A1) are substituted for. In other words, the substitution of the normalization constant $N^{a}$ in Eq. (33) gives the correct lattice-level fluctuationdissipation relation for all fluctuating densities $M^{a}, a \geq 4$. The amplitudes given in Eq. (33) recover the results of Ref. 26 for the standard integrator with $v_{s}^{2} / v_{c}^{2}=1 / 3$ when $q_{\mathrm{OU}}^{2}$ in lattice units is replaced by $1 / \tau^{2}$ (equivalent to a Taylor expansion of $q_{\mathrm{OU}}^{2}$ for small $\left.\Delta t / \tau\right)$ and $q_{\mathrm{FD}}^{2}$ is neglected (since in Ref. 26, they do not incorporate the noise into the equilibrium distribution). Thermalization of ghost densities is required as they drain fluctuations from the stresses [cf. Eq. (A6)] and they are also found in the source term [cf. Eq. (A4)] for the stresses (and vice versa), which shows explicitly the coupling between moments of different order at the local lattice level. Since it is not conserved, energy leaks into higher-order moments, which then dissipate it. If one were to use an energy-conserving LB scheme, ${ }^{40}$ this probably would not be needed. However, energy-conserving schemes require more than 15 moments in three dimensions. ${ }^{49}$

For $v_{s}^{2} \neq v_{c}^{2} / 3$, there are correlations in Eq. (4) between the diagonal stresses and they are not independent random variables. These correlations are determined by the nonzero diagonal $\eta_{\alpha \alpha \alpha \alpha}$ and off-diagonal elements $\eta_{\alpha \alpha \beta \beta}$ of the viscosity tensor of Eq. (3). For the equation of state $P_{\alpha \beta}$ $=P(\rho) \delta_{\alpha \beta}=\rho v_{s}^{2} \delta_{\alpha \beta}$, where $v_{s}$ is the speed of sound, we may calculate the elements using the formulae below Eq. (17) to be

$$
\begin{aligned}
& \eta_{\alpha \alpha \alpha \alpha}=2 \eta+\left(1-3 v_{s}^{2}\right) \eta=: 2 \eta+Y \eta ; \\
& \eta_{\alpha \alpha \beta \beta}=\left(1-3 v_{s}^{2}\right) \eta=Y \eta,
\end{aligned}
$$

where $\alpha \neq \beta$, and $\eta$ and $v_{s}^{2}$ are in units, where $\Delta x$ $=\Delta t=1$. Collecting all nine elements allows us to write down the correlation matrix $C$ whose matrix elements $C^{a b}=\left\langle\zeta^{a}(\mathbf{x}, t) \zeta^{b}\left(\mathbf{x}^{\prime}, t^{\prime}\right)\right\rangle, a, b \in\{4,5,6\}$ correspond to diagonal stresses computed from Eq. (4),

$$
\begin{aligned}
C & =\frac{q_{\mathrm{OU}}^{2}}{q_{\mathrm{FD}}^{2}}\left[\begin{array}{ccc}
\left\langle s_{x x}^{2}\right\rangle & \left\langle s_{x x} s_{y y}\right\rangle & \left\langle s_{x x} s_{z z}\right\rangle \\
\left\langle s_{x x} s_{y y}\right\rangle & \left\langle s_{y y}^{2}\right\rangle & \left\langle s_{y y} s_{z z}\right\rangle \\
\left\langle s_{x x} s_{z z}\right\rangle & \left\langle s_{y y} s_{z z}\right\rangle & \left\langle s_{z z}^{2}\right\rangle
\end{array}\right] \\
& =2 A_{\eta}\left[\begin{array}{ccc}
2+Y & Y & Y \\
Y & 2+Y & Y \\
Y & Y & 2+Y
\end{array}\right] .
\end{aligned}
$$

Corrections due to time discretization are contained in the same factor $A_{\eta}$ defined in Eq. (33). To construct random variables with this correlation matrix we factorize the correlation matrix $C$ for the diagonal components into Choleskydecomposed $^{50}$ form $C=2 A_{\eta} L L^{\mathrm{T}}$, where $\mathrm{T}$ denotes the transpose and

$$
L=\left[\begin{array}{ccc}
\sqrt{2+Y} & 0 & 0 \\
\frac{Y}{\sqrt{2+Y}} & \sqrt{\frac{2(2+2 Y)}{2+Y}} & 0 \\
\frac{Y}{\sqrt{2+Y}} & \frac{2 Y}{\sqrt{2(2+Y)(2+2 Y)}} & \sqrt{\frac{2(2+3 Y)}{2+2 Y}}
\end{array}\right] .
$$

We then generate a column of three independent Gaussian random variables and multiply them by $\sqrt{2 A_{\eta}} L$ to obtain the three correlated random variables that form the noise on the 
three diagonal elements of the stress tensor. Note that the required positive-definiteness of $C /\left(2 A_{\eta}\right)$ is not guaranteede.g., for our LB version of Eq. (3), its eigenvalues are 2, 2 , and $5-9\left(\partial P_{o} / \partial \rho\right)$, the last of which gives the criterion $v_{s}^{2}=\partial P_{o} / \partial \rho<5 / 9$ for the positive-definiteness of $C /\left(2 A_{\eta}\right)$ in lattice units. This limitation is shared not just by any standard, but also the multirelaxation time D3Q15 and D3Q19 models. ${ }^{44}$ The other random variables $(a>6)$ are the same as for the $v_{s}^{2}=v_{c}^{2} / 3$ case discussed above.

\section{RESULTS}

We shall first discuss the performance of our fluctuating LB fluid without immersed particles. As the total mass and momentum are conserved, it is a priori certain that local fluctuations in density and momentum must amount to zero when summed over the whole lattice. It is, therefore, interesting to see what happens to the temperature at intermediate length scales, which is not self-evident. We demonstrate how thermalization of center-of-mass (CM) degrees of freedom of the total system may be used to adjust the temperature at the whole system level. The second aspect we address is a coarsegrained polymer chain in bulk solution for which we perform standard static and dynamic measurements.

Unless otherwise specified, we have set the fluid parameters to those of water. The speed of sound is $v_{s}$ $=1497 \mathrm{~m} \mathrm{~s}^{-1}$, shear viscosity $\eta=1.0 \mathrm{~g} \mathrm{~m}^{-1} \mathrm{~s}^{-1}$, density $\rho$ $=998.2 \mathrm{~kg} \mathrm{~m}^{-3}$, and temperature $T=300 \mathrm{~K}$. The parameters are scaled to be multiples of $\Delta x=1.0 \mathrm{~nm}, \Delta t=0.3 \mathrm{ps}$, and $m_{\mathrm{LJ}}=0.001554 \mathrm{ag}$ (attogram) in the simulations. The speed of sound is, therefore, $v_{s} \approx \sqrt{0.2} v_{c}\left(v_{c}=\Delta x / \Delta t\right)$, for which $\Lambda=1.07 \mathrm{~g} \mathrm{~m}^{-1} \mathrm{~s}^{-1}$, which is smaller (by about a factor of 4) than the measured bulk viscosity. ${ }^{51}$

\section{A. Fluid temperature}

Temperature in the pure LB fluid without any immersed particles was assessed previously by Adhikari et al. ${ }^{25}$ by taking a Fourier transform of the local momenta $j_{\alpha}(\mathbf{x}, t)$ as

$$
\delta j_{\alpha}(\mathbf{k}, t) \equiv \frac{1}{\sqrt{V}} \sum_{\mathbf{x}} e^{i \mathbf{k} \cdot \mathbf{x}} \delta j_{\alpha}(\mathbf{x}, t),
$$

where $\delta j_{\alpha}(\mathbf{x}, t)=\rho(\mathbf{x}, t) u_{\alpha}(\mathbf{x}, t)-\left\langle\rho u_{\alpha}\right\rangle$ and the sum runs over all lattice sites in the system. Since $j_{\alpha}(\mathbf{x}, t)$ are real valued, only half of the Fourier components are independent and it suffices to plot the time-averaged quantity,

$$
T(\mathbf{k})=\left\langle|\delta \mathbf{j}(\mathbf{k}, t)|^{2}\right\rangle_{t} /\left(3 k_{\mathrm{B}} \rho_{0}\right),
$$

for $k \Delta x \in(0.2,3.0)$ as was done in Refs. 25 and 36. We plot $T(\mathbf{k})$ in Fig. 1(a). Two features are present: First, $T(\mathbf{k}=\mathbf{0})$ is zero due to conservation of total momentum. Second, $T(\mathbf{k}$ $>0$ ) deviates from the target value by no more than $2 \%$.

The impact of $T(\mathbf{k}=\mathbf{0})$ in a finite-sized system is worth considering further. As already mentioned, $T(\mathbf{k}=\mathbf{0})$ is due to the fact that the CM velocity of the system $\mathbf{V}$ is zero as the simulation box is stationary. One can consider a scenario where the simulation box is embedded in a much larger (or infinite) bath of the same fluid. In this case, the simulation box itself would undergo Brownian motion inside the bath. This
Brownian motion of the CM coordinates could be modeled using a Langevin equation,

$$
M_{T} \frac{d V_{\alpha}}{d t}=-\chi M_{T} V_{\alpha}+\Gamma_{\alpha},
$$

where the total mass of the simulation box is $M_{T}=\rho L^{3}$ and the components of the CM velocity are $V_{\alpha}=M_{T}^{-1} \sum_{\mathbf{x}} \rho(\mathbf{x}) \Delta x^{3} u_{\alpha}(\mathbf{x})$. When the second moment of the random force $\Gamma_{\alpha}$ is related to the friction coefficient $\chi$ by the fluctuation-dissipation theorem of the Langevin equation,

$$
\left\langle\Gamma_{\alpha}\right\rangle=0 ; \quad\left\langle\Gamma_{\alpha}(t) \Gamma_{\beta}\left(t^{\prime}\right)\right\rangle=2 k_{\mathrm{B}} T \chi M_{T} \delta_{\alpha \beta} \delta\left(t-t^{\prime}\right),
$$

then the equipartition theorem is obeyed and we have

$$
\frac{1}{2} M_{T}\left\langle V_{\alpha}^{2}\right\rangle=\frac{1}{2} k_{\mathrm{B}} T \text {. }
$$

We detail the CM thermalization procedure at the end of Appendix B. A plot of $T(\mathbf{k})$ for such a system is constant for all $\mathbf{k}$ including $\mathbf{k}=0$.

Now consider the block-averaged temperature defined in Eq. (6). Separate the CM motion from the block-averaged velocity of a block of size $L_{s}^{3}$ to define

$$
W_{\alpha}^{s}=\frac{\sum_{\mathbf{x} \in L_{s}^{3}} \rho(\mathbf{x}) u_{\alpha}(\mathbf{x})}{M_{s}}-V_{\alpha},
$$

where $M_{s}=\sum_{\mathbf{x} \in L_{s}^{3}} \rho(\mathbf{x})$. The block-averaged temperature defined in Eq. (6) then gives

$$
\begin{aligned}
\frac{1}{2} k_{\mathrm{B}} T_{s} & =\frac{1}{2}\left\langle\frac{\left(M_{s}\left(W_{\alpha}^{s}+V_{\alpha}\right)\right)^{2}}{M_{s}}\right\rangle \\
& =\frac{1}{2}\left\langle M_{s}\right\rangle\left\langle\left(W_{\alpha}^{s}\right)^{2}\right\rangle+\frac{1}{2}\left\langle M_{s}\right\rangle\left\langle V_{\alpha}^{2}\right\rangle \\
& =\frac{1}{2}\left\langle M_{s}\right\rangle\left\langle\left(W_{\alpha}^{s}\right)^{2}\right\rangle+\frac{1}{2} \frac{\left\langle M_{s}\right\rangle}{M_{T}} k_{\mathrm{B}} T \\
& =\frac{1}{2}\left\langle M_{s}\right\rangle\left\langle\left(W_{\alpha}^{s}\right)^{2}\right\rangle+\frac{1}{2}\left(\frac{L_{s}}{L}\right)^{3} k_{\mathrm{B}} T,
\end{aligned}
$$

where we have assumed that fluctuations in $M_{s}$ can be decoupled from fluctuations in $W_{\alpha}^{s}$ and have used Eq. (39) in the second to last line. The second term in the equation is due entirely to the CM motion and is clearly nonzero. With $T(\mathbf{k})$ being constant for all $\mathbf{k}$ including $\mathbf{k}=0$ we would expect, and indeed find, that $T_{S}\left(L_{S}\right)$ is constant independent of $L_{s}$. The inescapable conclusion is that if we do not thermostat the CM velocity, the $T_{s}$ cannot be constant but should be the local temperature minus the CM term above. This is exactly what is found and demonstrated in Fig. 1(b).

If we place a complex object, such as a polymer with radius of gyration $R_{g}=L_{s}$, into our fluid and then ask about its center-of-mass diffusion, then the distinction between $T_{s}$ and $T\left(k=2 \pi / L_{s}\right)$ is important as the polymer will behave as if its $\mathrm{CM}$ is experiencing the block-averaged temperature $T_{s}$. As a result, in comparing the polymer's diffusion in systems of different size $L$ we need to either thermostat the CM motion or ensure that $L_{s} / L$ is small enough that deviations from the target temperature are small. In order to distinguish the effects of the local and CM noise, we will use only local noise in the 

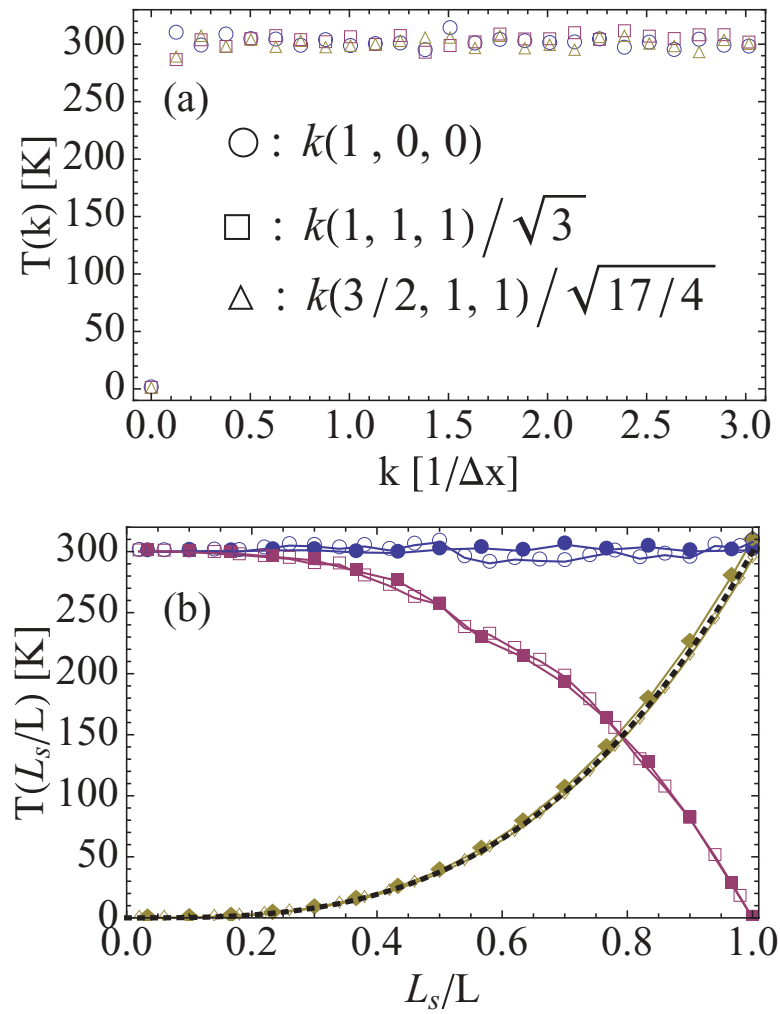

FIG. 1. (a) Temperature due to local stress fluctuations measured in a $L=50 \mathrm{~nm}$ box from Fourier-transformed local momentum fluctuations for three $\mathbf{k}$-vectors. Total momentum conservation can be seen as $T(\mathbf{k}=\mathbf{0})=0$. (b) Temperature measured from block-averaged momentum fluctuations using Eq. (6) as a function of ratio of subsystem size $L_{s}$ to linear size $L$ of the system (solid symbols: $L=30 \mathrm{~nm}$, hollow symbols: $L=50 \mathrm{~nm}$ ) with only local noise present in the stress tensor (squares), with noise only in the $\mathrm{CM}$ coordinate of the total system (diamonds), and with both local noise in the stress and Langevin thermostat on the CM coordinate present (circles). The dashed line is the function $300 \mathrm{~K}\left(L_{s} / L\right)^{3}$. The target temperature was $300 \mathrm{~K}$ and the speed of sound is $v_{s}=(1 / \sqrt{3})(\Delta x / \Delta t)$ in both (a) and (b).

remainder of this paper and use objects whose size is less than a quarter of the system size.

The higher-order density, $M_{\text {eq }}^{14}$ denoted as $K^{\text {eq }}$ in Eq. (18), may still be adjusted to tune the temperature. This is because this moment is related to damping of the stress fluctuations vis-à-vis Eq. (18). Figure 2 shows the effect of tuning the am-

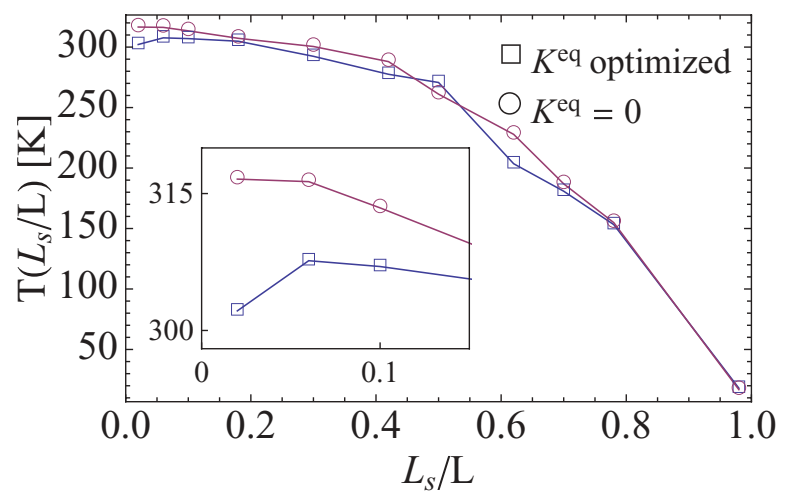

FIG. 2. The amplitude of the 14th moment can be adjusted to yield a flatter temperature profile at small length scales. This example is shown for our firstorder integrator and $v_{s}^{2}=0.1 \Delta x^{2} / \Delta t^{2}$. The squares and circles in Fig. 1 are also for the optimized $K^{\mathrm{eq}}$, but there $v_{s}^{2}=v_{c}^{2} / 3$.

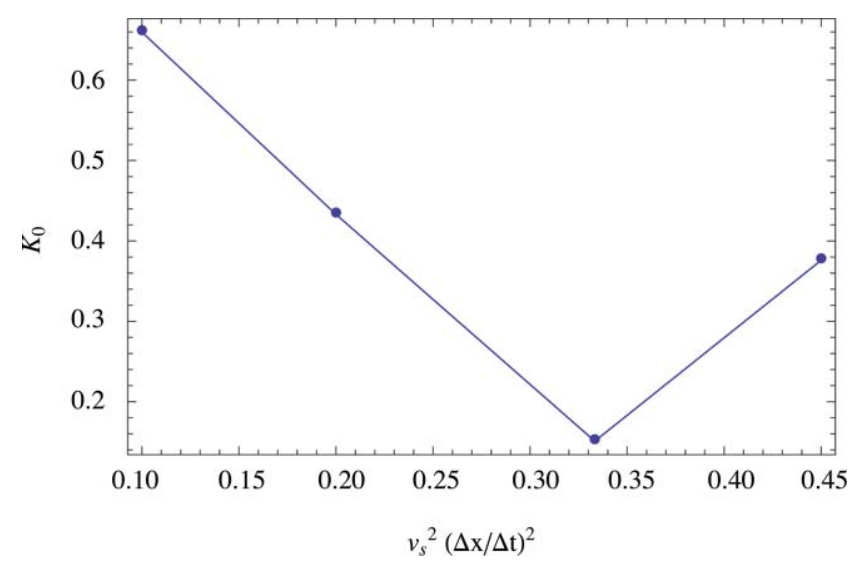

FIG. 3. The optimal values of the amplitude $K_{0}$ of the 14th equilibrium moment for the first-order integrator versus speed of sound squared. We found the function $0.15+2.18\left|(1 / 3)-\left(v_{s} / v_{c}\right)^{2}\right|$ a good fit to the data points. $K_{0}$ was optimized similarly for the other integrators giving $K_{0}\left(\left(v_{s} / v_{c}\right)^{2}\right.$ $<0.25)=1 / 3$ and $K_{0}\left(\left(v_{s} / v_{c}\right)^{2}>0.25\right)=1 / \sqrt{2}$ for the standard integrator with our noise and $K_{0}=\max \left\{3.8\left(1 / 3-\left(v_{s} / v_{c}\right)^{2}\right), 0\right\}$ for the second-order integrator.

plitude $K_{0}$ in Eq. (19), $K^{\mathrm{eq}}=K_{0}\left(\rho v_{c}^{2}-\operatorname{Tr}(P-s)\right)$ on temperature reproduction in the case of our first-order integrator for a case where $v_{s}^{2}=0.1 v_{c}^{2}$. The improvement of temperature reproduction is visible in the $L_{s}=\Delta x, \ldots, L / 4$ range where the optimized equilibria result in a flatter temperature profile, whereas the $K_{0}=0$ case has a monotonic decrease. The amplitude $K_{0}$ of the 14 th equilibrium density $\left[M_{\mathrm{eq}}^{14}\right.$ as per Eq. (9)] was set by minimizing the least squares error in the short length scale temperature $\left(L_{s} \leq L / 4\right)$ from the set point temperature of $300 \mathrm{~K}$ separately for each value of $v_{s}^{2}$ in the range $v_{s}^{2}=0.1, \ldots, 0.45 v_{c}^{2}$. This gave a set of optimal values $\left\{K_{0, j}\right\}$, which are plotted versus $v_{s}^{2}$ in Fig. 3 for the first-order integrator. $K_{0}$ was optimized similarly for the other integrators. One should note that even for $v_{s}^{2}=(1 / 3) v_{c}^{2}$, for which $\left(\rho v_{c}^{2}-\operatorname{Tr} P\right)=0$, there is a nonzero correction due to the fluctuations from $s$.

Our LB model and noise implementation also differ from previous works by the treatment of the pressure tensor $P_{\alpha \beta}$. In our model, the speed of sound need not be given by $v_{s}^{2}$ $=(1 / 3) v_{c}^{2}$ and $P_{\alpha \beta}$ can accommodate also off-diagonal components. This will make way for, e.g., studies of interface properties with fluctuations.

We next examine the temperature reproduction at various $v_{s}^{2}$, based on the temperature from Eq. (6) as a function of $L_{s} / L$ for the values $v_{s}^{2}=0.1, \ldots, 0.45 v_{c}^{2}$ comparing the standard integrator Eq. (30) and our first and secondorder integrators Eq. (27). The results are shown in Figs. 4(a) and $4(\mathrm{~b})$, and $4(\mathrm{c})$ for the $K_{0}$-optimized standard, firstand second-order integrators, respectively. For comparison, Fig. 4(d) contains our implementation of the fluctuating LB model by Dünweg et al. ${ }^{26}$ and our reproduction of Ladd's original results ${ }^{20}$ (both with $K^{\mathrm{eq}}=0$ and $M_{\text {eq }}^{13}=0$ ). For both of these we have Cholesky-decomposed the correlation matrix from the viscosity tensor corresponding to Eq. (35) although this decomposition is not mentioned in either work. The results are considerably worse for $v_{s}^{2} / v_{c}^{2} \neq 1 / 3$ if this is not done. In particular, Fig. 4(d) should be compared with 

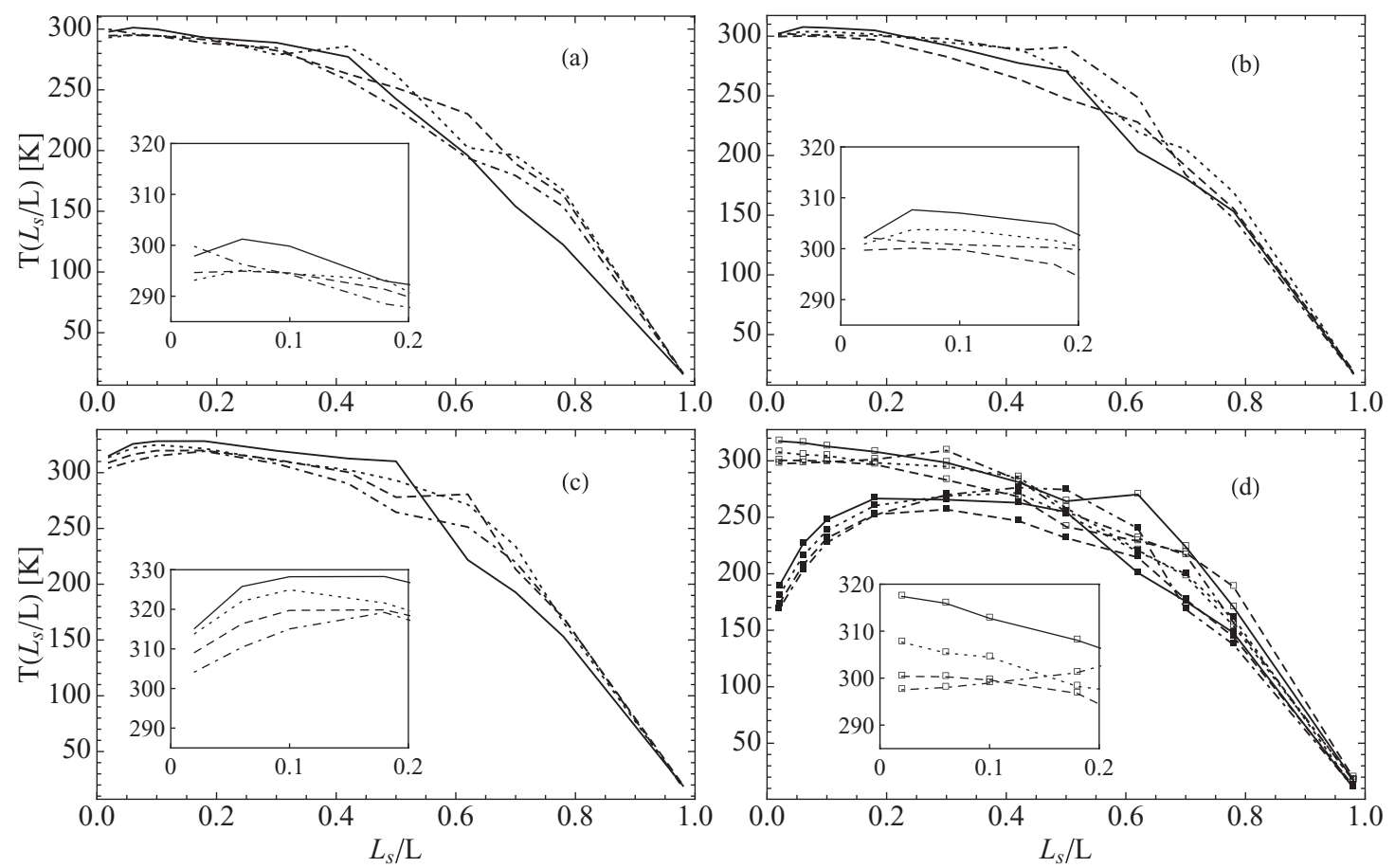

FIG. 4. Temperature measured in a $L^{3}=(50 \Delta x)^{3}$ box from velocity fluctuations as a function of linear size $L_{s}$ of the subsystem. The target temperature was $300 \mathrm{~K}$. Different curves correspond to different $v_{s}^{2}: 0.1$ (solid) 0.2 (dotted), $1 / 3$ (dashed) $0.45\left(\Delta x^{2} / \Delta t^{2}\right.$ ) (dotted-dashed). (a) standard integrator with our noise, (b) our first-order integrator with our noise, (c) our second-order integrator with our noise, and (d) our interpretation of Dünweg et al. (Ref. 26) ( $\square$ ) and our interpretation of Ladd's original scheme without ghosts ( $\mathbf{\square})$ (Ref. 20). The insets are close-ups of the corresponding upper left-hand corner. In particular, note the improvement in the reduced temperature spread in (a) over (d).

Fig. 4(a), for which the temperature spreads at $L_{s}<L / 5$ is smaller than that in $(\mathrm{d})$.

The $\left(v_{s} / v_{c}\right)^{2}=1 / 3$ case is somewhat more accurate for the Dünweg model probably because the noise amplitude and the standard integrator are derived with a consistent level of accuracy. Similarly, our first-order integrator of Eq. (27) has a consistent level of accuracy as the noise of Eq. (33) based on the SDE in continuum. The measured temperature profiles match nicely with the target temperature of $300 \mathrm{~K}$ at length scales $L_{s} \leq L / 4$. Also, the incorporation of the correlation matrix, Eq. (35), for the viscosity tensor makes the deviation between the data for different $v_{s}^{2}$ small. This would be very important for a nonideal equation of state, such as van der Waals, where the speed of sound is not constant, but in equilibrium, the temperature in the bulk of both phases should be the same. The fact that the temperature rises for the second-order [Fig. 4(c)] integrator at length scales slightly larger than that of the plaquette of the mesh is due to the second-order derivative terms in the integrator. They correlate the fluctuations between adjacent lattice sites. This offset is probably due to our neglect of some of the terms from Ito's lemma in the stochastic Taylor expansion of the equilibrium distribution. As the temperature is still fairly independent of length scale this integrator should still perform well. The main use of the second-order integrator is that it affords a large value for the coupling between the molecular dynamical solute and the LB fluid, which, as we shall see in the remaining sections, eliminates the need for external Langevin noise on the solute. If a particle phase is not of interest, the standard integrator [Fig. 4(a)] is a good choice.

\section{B. Single-particle diffusion}

An obvious requirement for a realistic description of hydrodynamic interactions is that an impermeable sphere of radius $a$ moving at a small velocity $\mathbf{v}$ relative to a still background fluid should experience a drag force equal to $6 \pi \eta a \mathbf{v}$ first derived by Stokes. ${ }^{53}$ Much effort has been dedicated to different ways of implementing the frictional fluid-particle interaction in simulations (see Refs. 30 and 34 and references therein). We have established criteria for consistent hydrodynamic radius of a solid inclusion in an LB solvent as presented in the accompanying paper. ${ }^{34}$ We found that by approximating the sphere by a set of $N_{v}$ nodes placed equivalently to a fullerene's structure at a distance $a_{n}$ about the sphere's center of mass, lattice corrugation effects were reduced significantly. Our scheme relies on Newton's equation of motion with the local frictional force,

$$
\mathbf{F}_{f}=-\gamma\left(\mathbf{v}(\mathbf{r}, t)-\mathbf{u}_{\mathrm{ip}}(\mathbf{r}, t)\right)
$$

where $\mathbf{u}_{\mathrm{ip}}(\mathbf{r}, t)$ is an interpolated fluid velocity at the particle location based on the flow field at nearby lattice sites and it acts as the source of fluctuations for the inclusion, and $\mathbf{v}$ is the particle velocity.

The fluctuating field $\mathbf{u}_{\mathrm{ip}}$ is thus a heat bath for the molecular dynamical objects on which the frictional force is exerted. Relevant to the present study, we showed that in our consistent scheme, Stokes drag, drag torque in shear flow, and hydrodynamic forces between particles depend strongly on the algorithmic (bare) coupling parameter $\gamma \cdot{ }^{34}$ We adjusted $\gamma$ so that consistency between these deterministic measures of hydrodynamic radius and theory was attained. We show here that 
for the very same value of $\gamma$, the fluctuation-dissipation theorem is also obeyed with thermal fluctuations implemented as in the current paper.

We emphasize the fact that $\gamma$ is neither arbitrary nor is it to be naively equated with $6 \pi \eta a_{n}$ as $\mathbf{u}_{\text {ip }}$ is not the far-field velocity as assumed in $6 \pi \eta a \mathbf{v}$, but it is adjusted to ensure consistency between different measures of hydrodynamic radius. ${ }^{34}$ A sufficiently large value $\gamma_{s}=\gamma_{s}\left(\rho, N_{v}, a_{n}\right)$ for $\gamma$ will make the radii based on the different measures all equal $a_{n}$. Similar, but not necessarily consistent schemes are cited in Ref. 34. Only at $\gamma=\gamma_{s}$ the particles experience the correct temperature, drag force, and obey the macroscopic fluctuation-dissipation theorem. Use of a different value of $\gamma$ would require additional thermostatting of the particle degrees of freedom to obtain the correct temperature.

To demonstrate the fluctuation-dissipation theorem of the algorithm, we study the velocity autocorrelation function VACF $=(1 / 3)\langle\mathbf{v}(t) \cdot \mathbf{v}(0)\rangle$. The equipartition theorem tells us that at $t=0$ the VACF should be $k_{\mathrm{B}} T / M$. With the assumption that the particle of hydrodynamic radius $R_{H}$ is well described in a continuum model with no-slip impenetrable boundary conditions, the VACF has been calculated from the linearized (compressible) Navier-Stokes equations in Refs. 54 and 55. The result is complex so we just summarize the relevant findings: at short times, $t<t_{s} \equiv R_{H} / v_{s}$, where $v_{s}$ is the speed of sound, sound waves generated by the particle motion carry away some of the particle's momentum resulting in a quick decay from $k_{\mathrm{B}} T / M$ at $t=0$ to $k_{\mathrm{B}} T / M^{*}$, where $M^{*}$ $=M+\rho 2 \pi R_{H}^{3} / 3$ (see Ref. 55). At longer times compressibility is not important and the incompressible NavierStokes equations can be used to predict the asymptotic longtime power-law tail ${ }^{33,55,56} k_{\mathrm{B}} T\left(12 \rho \sqrt{\pi^{3} \nu^{3}}\right)^{-1} t^{-3 / 2}$, where $v$ $=\eta / \rho$ is the kinematic viscosity. All of these effects have been observed in particle systems (see Ref. 57 for a recent example).

For the deterministic comparison of a particle coming to rest, we consider a particle dragged with constant external force $F_{0}$ until it reaches steady state (walls far from the particle are needed in a direction parallel to the particle motion in order for the fluid not to "catch up" with the particle). At $t>0$ the external force is removed and the particle and solvent relax toward a quiescent state. Iwashita et al. ${ }^{33}$ have shown that the fluctuation-dissipation theorem implies that $\left(-1 / F_{0}\right) \mathrm{d} v(t) / \mathrm{d} t$ for this relaxation of the particle velocity is equal to the VACF divided by $k_{\mathrm{B}} T$ in an incompressible fluid. This should be the same as the compressible case for $t>t_{s}$ (the time during which sound waves affect the VACF) but should go to $k_{\mathrm{B}} T / M^{*}$ at $t=0$ as there will not be any sound waves generated by this protocol (starting from steady state), even in the compressible case.

The VACF and the comparison to the deterministic relaxation is shown in Fig. 5. The particle had a hydrodynamic radius of $2.7 \Delta x(\Delta x=10 \mathrm{~nm}$ and $L=32 \Delta x)$ for which the sound time $t_{s}$ is $4.7 \Delta t$. We see perfect agreement for times $t>t_{s}$ between the deterministic relaxation and the VACF as predicted by the fluctuation-dissipation theorem. We also see excellent agreement with the asymptotic $t^{-3 / 2}$ behavior. We have left the $1 / M$ factor out of the curve (which would have given an intercept of one on

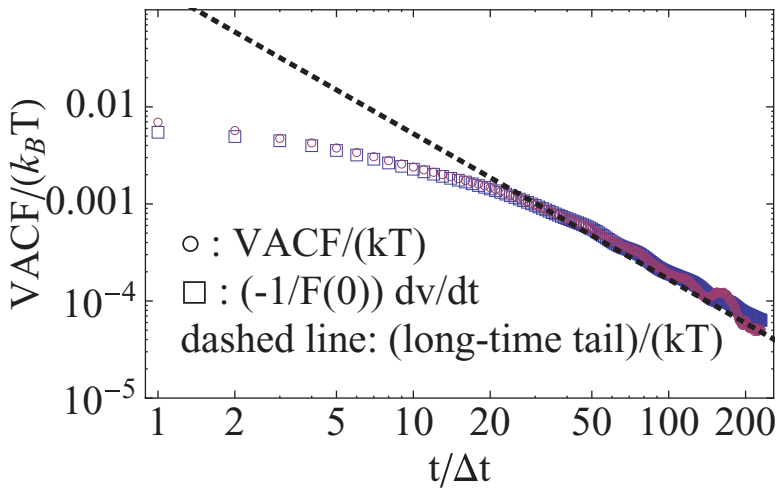

FIG. 5. VACF of a particle diffusing in the LB fluid with thermal fluctuations ( $\square$ ) and the rate of decay of the velocity of a particle that was in steady state motion due to a constant force $F_{0}$ for $t<0$ and then relaxes to a quiescent state when the force is removed for $t>0$ in a LB fluid with no thermal fluctuations. The dashed line is the asymptotic long-time power-law tail, $k_{\mathrm{B}} T\left(12 \rho \sqrt{\pi^{3} v^{3}}\right)^{-1} t^{-3 / 2}$, divided by $k_{\mathrm{B}} T$ (Ref. 33).

the $y$-axis) so that the intercept of the VACF curve gives $1 / M$ and that of the deterministic curve gives $1 / M^{*}$. This gives $M=150 \mathrm{ag}$ and $M^{*}=190 \mathrm{ag}$. The difference $M^{*}-M$ $=\rho 2 \pi R_{H}^{3} / 3$ expected from Zwanzig's calculation ${ }^{55}$ can be used to give one measure of $R_{H}$ for the particle $(27 \mathrm{~nm})$. This can be used to predict $M$ which is the mass of the fullerene shell we used to construct our particle (66.9 ag) and the fluid that necessarily always moves with it (i.e., fluid inside its hydrodynamic radius). This predicts $M=149$ ag which is very close to the $M=150$ ag measured. Thus we can conclude that both the fluctuation-dissipation theorem and equipartion $(t=0$ prediction) are well obeyed.

Measuring the diffusion coefficient has been used in the past as a test of polymer dynamics in an LB solvent. ${ }^{30,31} \mathrm{To}$ measure the diffusion constant of a single particle we place it in a cubic simulation box with periodic boundary conditions. To measure the diffusion coefficient in three dimensions we track the mean squared displacement as a function of time and use the relation, ${ }^{58}$

$$
D=\lim _{\left|t-t^{\prime}\right| \rightarrow \infty} \frac{\left\langle\left(\mathbf{r}(t)-\mathbf{r}\left(t^{\prime}\right)\right)^{2}\right\rangle}{6\left(t-t^{\prime}\right)} .
$$

Rather than using an infinite time displacement, we look at the slope of $\left\langle\left(\mathbf{r}(t)-\mathbf{r}\left(t^{\prime}\right)\right)^{2}\right\rangle$ versus $6\left(t-t^{\prime}\right)$ which eventually becomes constant (equal to $D$ ).

The one remaining caveat is that there are also finite-size effects in the measurement of the diffusion coefficient. ${ }^{59,60}$ That is, we expect

$$
D(L)=\frac{k_{\mathrm{B}} T}{6 \pi \eta R_{H}}-\frac{k_{\mathrm{B}} T}{6 \pi \eta} \frac{B}{L},
$$

where $B$ is a dimensionless numerical constant with the theoretical prediction of 2.837 in the zero Reynolds number limit calculated in Ref. 60 from the Oseen tensor, and $R_{H}$ is a hydrodynamic radius. Thus, a plot of $D R_{H}$ versus $R_{H} / L$ should give a straight line onto which data from different radii should fall. Such a plot is shown in Fig. 6 which follows the prediction of Eq. (42). $R_{H}$ is slightly larger than the radius of the fullerene shell used to construct our particle but can be determined independently by drag force or torque measurements. 


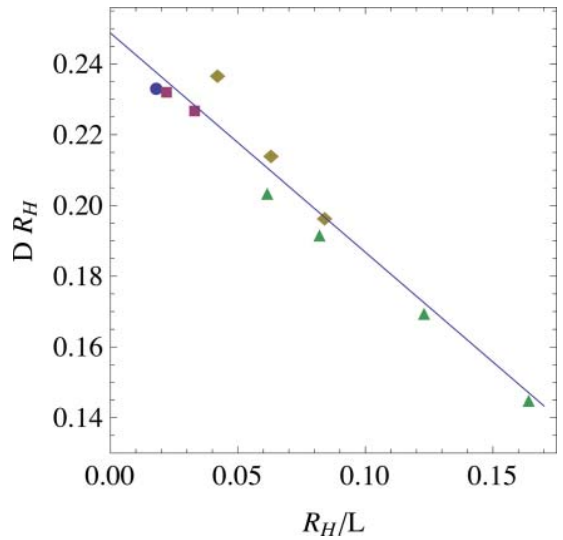

FIG. 6. Diffusion coefficient $D$ as a function of the hydrodynamic radius $R_{H}$ and linear system size $L$. Different symbols are from runs with particles of different radii. A linear fit gives 2.82 for the numerical constant $B$ in Eq. (42).

In addition, the theoretical approximation of 2.837 is in excellent agreement with the fit parameter $B=2.82 \pm 0.1$ of Eq. (42). Thus, not surprisingly given the relation between the diffusion constant and the VACF, the particle also obeys the macroscopic fluctuation-dissipation relation of Eq. (42).

\section{Polymer in fluid}

There are several well-established results in polymer physics for the static and dynamic behaviors of a single polymer chain in bulk solution. ${ }^{61}$ They provide a suitable benchmark to see how our fluctuating fluid with an immersed polymer chain fares against theoretic predictions and other models. We use the finitely extensible nonlinear elastic (FENE) chain $^{62}$ to model a polymer as $N$ beads interconnected by $N-1$ springs. The excluded-volume effect is captured by the Lennard-Jones potential. These interactions are written as

$$
\begin{aligned}
U\left(\mathbf{r}_{i}, \mathbf{r}_{j}\right)= & -\frac{1}{2} k R_{0}^{2} \log \left(1-\frac{r_{i j}^{2}}{R_{0}^{2}}\right) \delta_{|j-i|, 1} \\
& +4 \epsilon\left(\left(\frac{\sigma}{r_{i j}}\right)^{12}-\left(\frac{\sigma}{r_{i j}}\right)^{6}+\frac{1}{4}\right) \Theta\left(2^{1 / 6}-\frac{r_{i j}}{\sigma}\right),
\end{aligned}
$$

where $r_{i j}=\left|\mathbf{r}_{i}-\mathbf{r}_{j}\right|$, the first term is the FENE potential tying consecutive beads together in the chain, $\Theta(x)$ is the Heaviside step function and the second term is the repulsive truncated and shifted 12-6 Lennard-Jones potential. The parameters of the model are the spring constant $k=30 \epsilon \sigma^{-2}$, the maximum extension of a bond $R_{0}=1.5 \sigma$, the energy scale $\epsilon=k_{\mathrm{B}}(300 \mathrm{~K})$, where $k_{\mathrm{B}}$ is the Boltzmann constant, and the length scale $\sigma=\Delta x=1 \mathrm{~nm}$. The monomers follow Newton's equation of motion,

$$
m \ddot{\mathbf{r}}_{i}=-\sum_{j \neq i} \nabla_{i} U\left(\mathbf{r}_{i}, \mathbf{r}_{j}\right)+\mathbf{F}_{f i}\left(\mathbf{r}_{i}, \mathbf{v}_{i}, \mathbf{u}\left(\mathbf{r}_{i}, t\right)\right),
$$

where the particle mass $m=m_{\mathrm{LJ}}$ is the inertia needed in defining the Lennard-Jones timescale $\tau_{\mathrm{LJ}}=\sqrt{m \sigma^{2} / \epsilon}$ of the model. The second term on the right-hand side is a local force to describe frictional fluid-particle interaction,

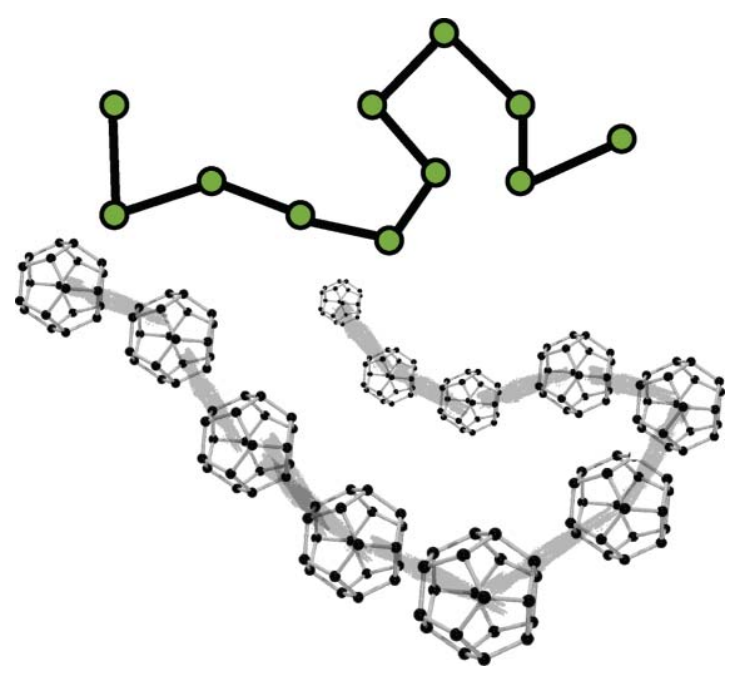

FIG. 7. A schematic picture of a point-particle chain, for which lattice corrugation effects are large and the hydrodynamic radius is not well defined in the LB scheme (top). A chain consisting of composite beads of 20 nodes each (bottom). The simulations were run using beads of 60 nodes and a strength of coupling $\gamma$ large enough to guarantee a consistent hydrodynamic radius (see Ref. 34 for detailed analysis).

$$
\mathbf{F}_{f i}=-\gamma\left(\mathbf{v}_{i}\left(\mathbf{r}_{i}, t\right)-\mathbf{u}_{\mathrm{ip}}\left(\mathbf{r}_{i}, t\right)\right),
$$

where $\mathbf{u}_{\mathrm{ip}}$ is an interpolated fluid velocity at the particle location based on the flow field at nearby lattice sites.

Ahlrichs and Dünweg ${ }^{30}$ simulated a FENE polymer made of point particles, cf. Fig. 7, in a fluctuating LB fluid at $v_{s}^{2}=(1 / 3)\left(\Delta x^{2} / \Delta t^{2}\right)$ with additional Langevin noise applied independently to the beads. As described in Ref. 29, they observed that fluctuations transmitted from the fluctuating LB fluid to a tracer point particle undergoing Brownian motion did not result in a consistent temperature as measured from the expected equipartition relation $3 k_{\mathrm{B}} T=m\left\langle\mathbf{v}^{2}\right\rangle$ for the particle velocity, but $\left\langle\mathbf{v}^{2}\right\rangle$ turned out to be a function of $\gamma$. To obtain a uniform temperature profile independent of $\gamma$, they added Langevin noise to the particle and subtracted the very same noise locally from the fluid to conserve momentum.

While their approach works for a single point particle, the added Langevin noise alters hydrodynamic correlations between any degrees of freedom coupled through the momentum-conserving fluid and the internal structure of the molecular dynamical object. In this paper, we study a polymer made out of rigid composite beads, see bottom of Fig. 7, and allow it to bask in the heat bath provided by the fluctuating fluid alone. Since we have already shown that a single extended or point particle obeys the fluctuation-dissipation theorem when $\gamma=\gamma_{s}$ in the previous subsection, here we shall concentrate on testing a polymer chain suspended in the LB fluid.

To speed up the polymer dynamics for these tests, we decrease the density to $1 / 60$ of that of water, increase the temperature to $310 \mathrm{~K}$ and to boost hydrodynamic interactions we multiply the kinematic viscosity by a factor of 1.4 . We refer to these as "relaxed parameters." The FENE polymer is then constructed from composite particles of $N_{v}=60$ nodes, which is considerably more than necessary, but MD is not a big fraction of total computational cost in these simulations. A 
monomer's radius is set to $a_{n}=0.6 \mathrm{~nm}$ and its mass is set by requiring neutral buoyancy, i.e., $m_{\mathrm{LJ}}=(4 / 3) \pi a_{n}^{3} \rho$. In order to have a consistent hydrodynamic radius for each monomer, ${ }^{34}$ the value of $\gamma N_{v}$ is set to be $\gamma_{s} N_{v} \approx 1.62 \mathrm{pg} \mathrm{ns}^{-1}$.

The effective Schmidt number for a spherical particle of radius $0.6 \mathrm{~nm}$ in water is $S c=(\eta / \rho) /\left(k_{\mathrm{B}} T /(6 \pi a \eta)\right)$ $\approx 2740$, which is six times larger than the value of 460 for water ${ }^{17}$ calculated using the self-diffusion coefficient of a water molecule. We resort to this calculation as the self-diffusion coefficient of the LB fluid is not well defined. We get $S c \approx 90$ for the same sphere using our relaxed parameters, but the self-diffusion coefficient of the LB fluid is reduced correspondingly. The difference in $S c$ computed in the two different ways is important as that indicates slower dynamics for the particle phase than for the solvent. The Péclet number for the same sphere in water can be approximated as $P e=\sqrt{\left\langle\mathbf{v}^{2}\right\rangle} a /\left(k_{\mathrm{B}} T /(6 \pi \eta a)\right)$ $=6 \sqrt{3} \pi a^{2} \eta / \sqrt{m_{\mathrm{LJ}} k_{\mathrm{B}} T} \approx 190$, which decreases to 34 for our relaxed parameters.

\section{Static polymer scaling}

The size of a polymer is characterized by its radius of gyration $R_{g}$,

$$
\left\langle R_{g}^{2}\right\rangle=\frac{1}{N} \sum_{m}\left\langle\left(\mathbf{r}_{m}-\mathbf{r}_{\mathrm{cm}}\right)^{2}\right\rangle
$$

where the subscript $\mathrm{cm}$ refers to the center of mass of the chain. $R_{g}$ is expected to scale as a function of the degree of polymerization $N$ as

$$
\left\langle R_{g}\right\rangle \sim N^{v},
$$

where $v$ is the Flory exponent that accounts for the excludedvolume effect. Its asymptotic value for a self-avoiding random walk in $3 \mathrm{D}$ is $v \approx 0.5877 .{ }^{63}$ We have verified Eq. (47) for our coupled LB-MD model by measuring $R_{g}$ and fitting the data to the functional form of $a N^{b}$ with the result $R_{g}=(0.49 \sigma) N^{0.61}$ using data for $N=48,64,80$, and 96 .

The Flory exponent may be obtained independently from the angularly averaged static structure factor $S(k)$ defined through the monomer density function $\rho(\mathbf{r})=\sum_{m} \delta\left(\mathbf{r}-\mathbf{r}_{m}\right)$ and its Fourier transform $\hat{\rho}(\mathbf{k})=\mathcal{F}(\rho(\mathbf{r}))=\sum_{m} \exp \left(i \mathbf{k} \cdot \mathbf{r}_{m}\right)$ as

$$
\begin{aligned}
S(k) & =\frac{1}{N} \frac{1}{4 \pi} \int\left\langle\hat{\rho}(\mathbf{k}) \hat{\rho}^{*}(\mathbf{k})\right\rangle \mathrm{d} \Omega \\
& =\frac{1}{N} \sum_{m, n=1}^{N}\left\langle\frac{\sin \left(k\left|\mathbf{r}_{m}-\mathbf{r}_{n}\right|\right)}{k\left|\mathbf{r}_{m}-\mathbf{r}_{n}\right|}\right\rangle .
\end{aligned}
$$

The static structure factor should follow a scaling relation for a range of $k$ values,

$$
S(k) \sim k^{-1 / \nu} \text {. }
$$

We plot $S(k)$ for the chain length-system size ratios $N / L=32 / 36,48 / 46,64 / 56,80 / 64$, and 96/72 in Fig. 8 using a logarithmic scale. We also show the corresponding running slopes of logarithms to locate the scaling region that should show up as a constant region $(\partial \log [S(k)] / \partial \log k$ $=(-1 / v))$. We find a broad flattening of the curves that shows
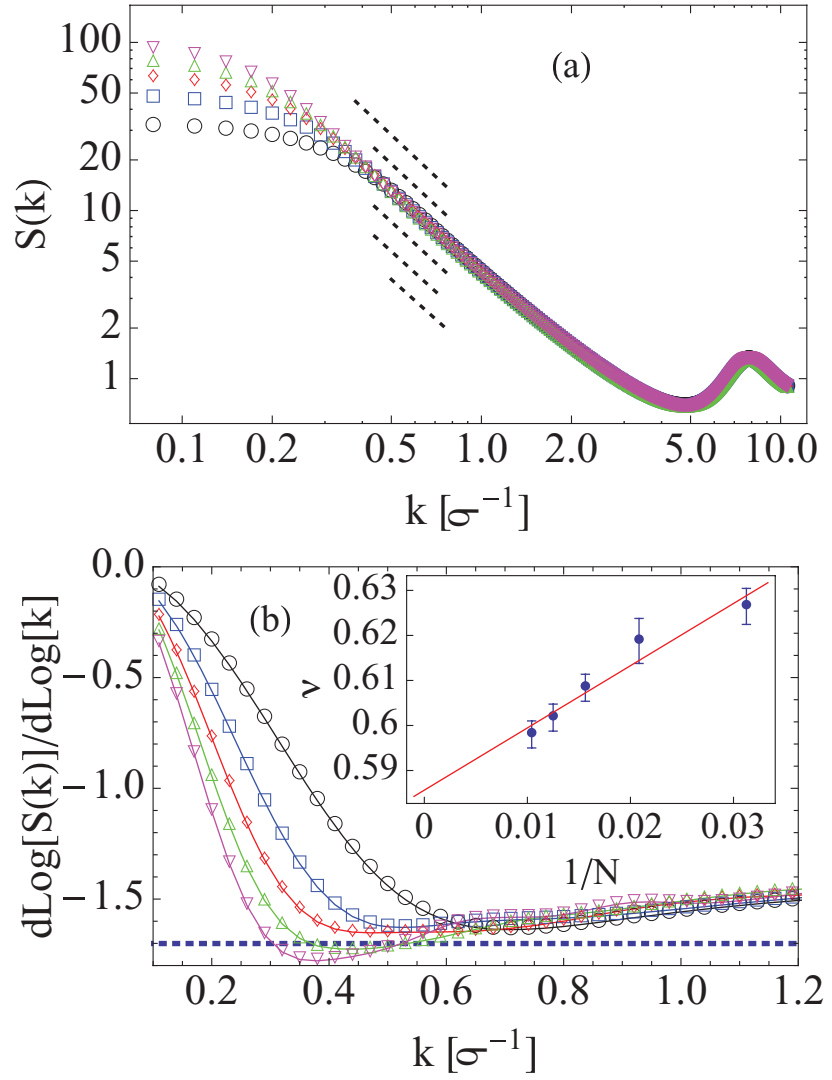

FIG. 8. (a) Static structure factor for $N=32(\circ), 48(\square), 64(\diamond), 80(\triangle)$, and $96(\nabla)$. The black dotted lines indicate the corresponding fitting/scaling regions determined from (b). The scaling region broadens toward smaller $k$ values as the chain gets longer. (b) The slope of the logarithm of $S(k)$ vs $k$ used to extract the scaling region and a slope, where a plateau equal to $-1 / v$ should develop. The horizontal dotted line indicates the theoretic value of $-1 / v=-1.70$. The inset is a finite-size scaling plot that gives $v=0.586 \pm$ 0.005 in the $N \rightarrow \infty$ limit. Errors in the main plots are smaller than the size of symbols.

signs of saturation at the theoretic value of $-1 / v=-1.70$ for chains $N=80$ and 96 . We have also extracted the asymptotic value of $0.586 \pm 0.005$ for $v$ through finite-size scaling in the inset of Fig. 8(b). The error in $v$ as a function of $1 / N$ was estimated by including a broader range of $k$ values in fitting and seeing the effect on $v$ as a function of $1 / N$ [the range of $k$ used for the mean values of $v$ are indicated as dashed lines in Fig. 8(a)]. The ratio $L / R_{g}$ was kept constant at about 9.5 in our simulations.

We also examined the bond-bond correlation function along the backbone of the chain defined as

$$
\left\langle\cos \theta_{m, m+n}\right\rangle_{m} \equiv\left\langle\frac{\mathbf{r}_{m+1}-\mathbf{r}_{m}}{\left|\mathbf{r}_{m+1}-\mathbf{r}_{m}\right|} \cdot \frac{\mathbf{r}_{m+n+1}-\mathbf{r}_{m+n}}{\left|\mathbf{r}_{m+n+1}-\mathbf{r}_{m+n}\right|}\right\rangle_{m} .
$$

We compare the measurements from our LB simulations to runs where the LB fluid term in the monomer's equation of motion is replaced by the commonplace Langevin thermostat and the composite monomers are replaced by a point particle of equal mass. The damping factor in the frictional term of the Langevin equation is set to $1.4 \tau_{\mathrm{LJ}}$. We find in Fig. 9(a) a close correspondence in the bond-bond correlation between LB chains of length $N \geq 64$ and Langevin chains of length $N \geq 128$. The close correspondence for $n=5-25$ 

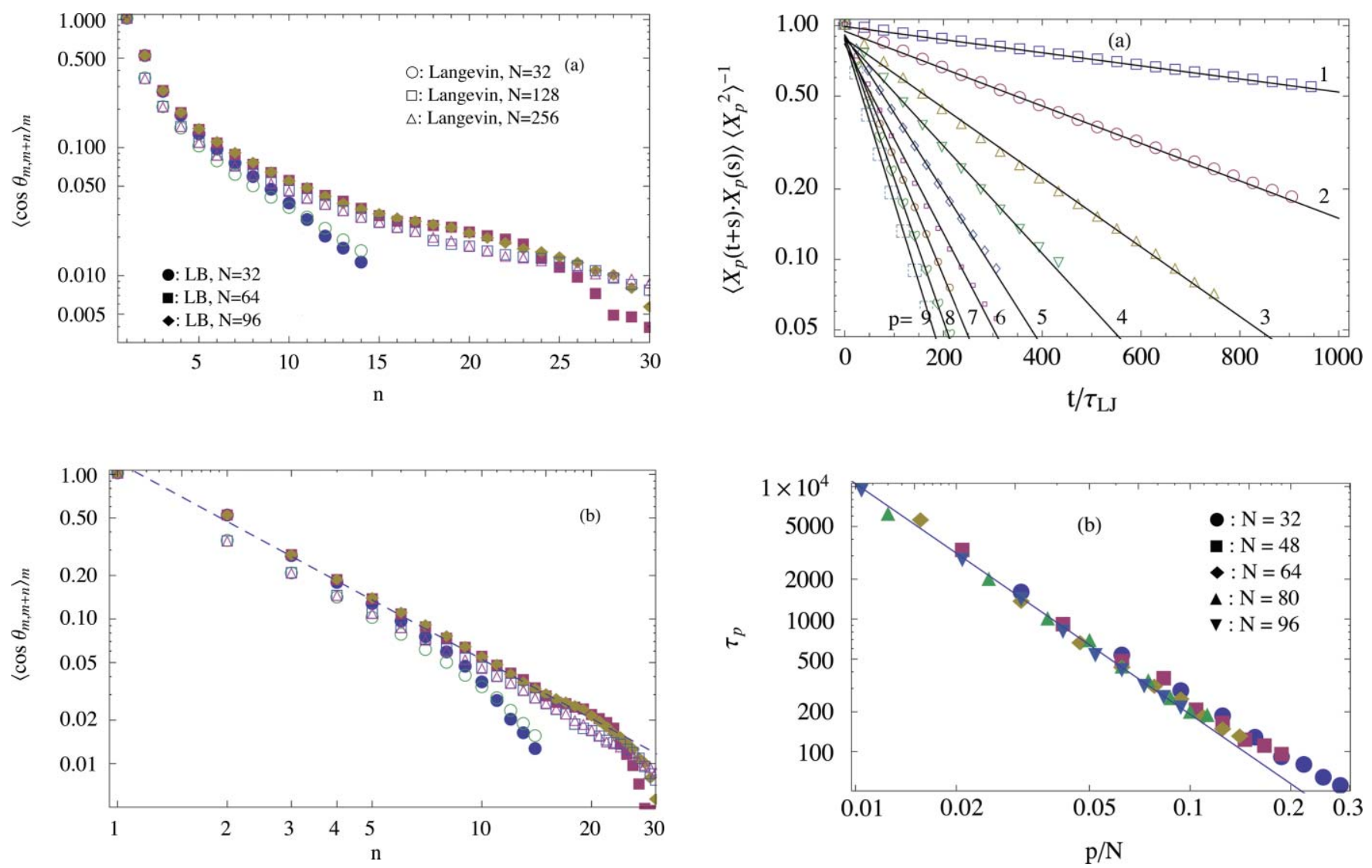

FIG. 9. Bond angle correlation function for a 32-bead chain as a function of separation of monomers along the chain backbone (see text). The filled symbols are data from LB runs and hollow symbols are from corresponding Langevin runs. The power law $\sim n^{-1.36 \pm 0.01}$ fits well both to the LB and Langevin data indicating that the chain is properly thermalized in the LB solvent when the strength of coupling $\gamma$ equals $\gamma_{s}$ of Eq. (45) for which a consistent hydrodynamic radius is obtained (Ref. 34).

indicates that the LB chain is properly thermalized as the decay is temperature dependent. We observe also that an increased local exclusion ensues in the LB solvent, which is seen as the slower initial decay of the function for $n=2-5$, i.e. the effective size of the beads is slightly larger in the full LB simulation. Panel (b) in Fig. 9 shows the same data in $\log -\log$ scale with a fit $n^{-1.36}$ to the LB data in the range $n=3-15$ for $N=64$ and 96 and $n=3-5$ for $N=32$, and we find the slope in the corresponding Langevin data to be identical. This suggests that hydrodynamics has no effect on such an equilibrium property in agreement with the findings in Ref. 64 . The chains we simulated are short compared to those in Ref. 65 , where chains of length $N=400, \ldots, 6400$ modeled as self-avoiding walks (SAW) on a simple cubic lattice were observed to scale as $n^{-0.824}$ and the same SAWs at the $\theta$-temperature as $n^{-1.5}$, but our tentative power law is reasonable as it suggests good-solvent conditions.

\section{E. Polymer dynamics}

\section{Rouse mode analysis}

We have done a standard Rouse mode analysis for our chain. The Rouse modes are written as ${ }^{66}$

FIG. 10. (a) Rouse mode correlation functions $p=1-9$ for $N=32$ in $\log -$ linear scale with fits for extraction of relaxation times $\tau_{p}$. (b) Relaxation times vs the ratio of mode number $p$ to degree of polymerization $N$. The slope of the fit in (b) equals $z v=1.74 \pm 0.01$, which gives $z \approx 2.97 \pm 0.04$, when the asymptotic value $v=0.586$ from Fig. $8(b)$ is used.

$$
\mathbf{X}_{p}=\frac{1}{N} \sum_{m=1}^{N} \mathbf{r}_{m} \cos \left(\frac{p \pi}{N}\left(m-\frac{1}{2}\right)\right),
$$

where $p=1,2, \ldots, N-1$ is the mode number. For the Zimm model, these modes should decay exponentially with a relaxation time $\tau_{p}$ according to

$$
\left\langle\mathbf{X}_{p}(t+s) \cdot \mathbf{X}_{p}(s)\right\rangle_{s}=\left\langle\mathbf{X}_{p}^{2}\right\rangle e^{-t / \tau_{p}} .
$$

We do not expect our LB model to produce a one-to-one mapping to Zimm theory as there is no Langevin-type noise on the monomers that would dominate the heat bath provided by the fluctuating fluid and make the mode-coupling matrix diagonal. Nevertheless, it is insightful to compare the measured relaxation times to those from theory that are expected to scale as ${ }^{67}$

$$
\tau_{p}^{-1} \sim\left(\frac{p}{N}\right)^{z \nu} r_{v}(p)
$$

where $z$ is the dynamic scaling exponent that depends on chain rigidity, $v$ is again the Flory exponent, and $r_{v}(p)$ is a slowly varying function of $p$ that arises when the summation in Eq. (A1) is approximated by an integral. Its form and detailed discussion is well presented in Refs. 30 and 67. We measured the relaxation times $\tau_{1}$ to $\tau_{9}$ for $N=32,48$, 64,80 , and 96 by performing a linear fit to the logarithm of Eq. (A1). An example of such fits is shown in Fig. 10(a). 
When the relaxation times data for all the chains are plotted in log-log representation simultaneously [Fig. 10(b)], the data fall onto a line with the exponent $z v=1.74 \pm 0.01$ for $p / N=0.01, \ldots, 0.1$. The correction $r_{v}(p)$ does not improve the data collapse in Fig. 10(b) as was also stated in Ref. 64 where different Rouse modes for a fixed $N$ were collapsed onto a single line. By dividing the fitted exponent $z v$ by the asymptotic Flory exponent $v=0.586 \pm 0.005$ from Fig. 8(b), we obtain a value of $2.97 \pm 0.04$ for $z$ in agreement with $z=3$ in the Zimm model.

\section{Chain diffusion}

Dynamic polymer scaling in the dilute limit is built on the relations of Eq. (47), the scaling relation for $R_{g}$, and $D_{\mathrm{cm}}$ $\sim N^{-v_{D}}$, which relates the center-of-mass diffusion coefficient, $D_{\mathrm{cm}}$, to its scaling exponent $v_{D}$. In the present context, the formula relating $D_{\mathrm{cm}}$ to the mean-squared displacement, ${ }^{58}$

$$
D_{\mathrm{cm}}=\lim _{\left|t-t_{0}\right| \rightarrow \infty} \frac{\left\langle\left(\mathbf{r}_{c m}(t)-\mathbf{r}_{c m}\left(t_{0}\right)\right)^{2}\right\rangle}{6\left|t-t_{0}\right|},
$$

will prove useful.

As any simulation is performed in a finite system, it is important to account for finite-size effects. Several works ${ }^{59,60,68}$ report an expression of the form,

$$
D_{\mathrm{cm}}=\frac{k_{\mathrm{B}} T}{6 \pi \eta}\left(\frac{A}{R_{g}}-\frac{B}{L}\right),
$$

where $A$ and $B$ are dimensionless constants and $L$ is the linear size of the system. We expect our polymer model to yield congruous results for $B$ to our earlier work dealing with a single colloid in Subsection IV B.

A finite-size scaling analysis for short chains of 16, 24, and 32 beads, Fig. 11(a), confirms the $1 / L$ scaling. The fits give an average value of $2.8 \pm 0.05$ for $B$, with which the analytic approximation of 2.837 of Ref. 60 valid for the Oseen tensor agrees well. The calculation in Ref. 60 for $B$, which has been performed in the Ewald representation, ${ }^{69}$ is, to our knowledge, the most accurate in the Oseen limit as it accounts for the periodic images.

Since a polymer is not a solid sphere of radius $R_{g}, A$ is necessarily larger than unity. Dünweg et $a l .{ }^{70}$ have done extensive work on measuring $\left\langle R_{g} / R_{I}\right\rangle$ with the result 1.63 \pm 0.01 for a bead-spring model in continuum space (in this case they use $R_{I}$ as the static hydrodynamic radius defined through $\left.\left\langle 1 / R_{I}\right\rangle=\sum_{m \neq n}\left\langle 1 /\left|\mathbf{r}_{m}-\mathbf{r}_{n}\right|\right\rangle\right)$. Sunthar and Prakash $^{71}$ have pointed out that the static measure $R_{I}$ is systematically larger than the dynamic hydrodynamic radius present in Eq. (A1) as $R_{H}=R_{g} / A$. We have extracted $A$ $=R_{g} / R_{H}$ from the diffusion data and plotted it as a function of $R_{g}$ in Fig. 11(b). We used the mean value of $B=2.8$ to extract $A$ for the chains for which we did not have data as a function of system size. We find that $A$ approaches $1.67 \pm 0.05$ asymptotically, which is close to Dünweg and co-workers' result.

We conclude, as for the single-particle diffusion, our polymer obeys the fluctuation-dissipation theorem with no added adjustable parameters.
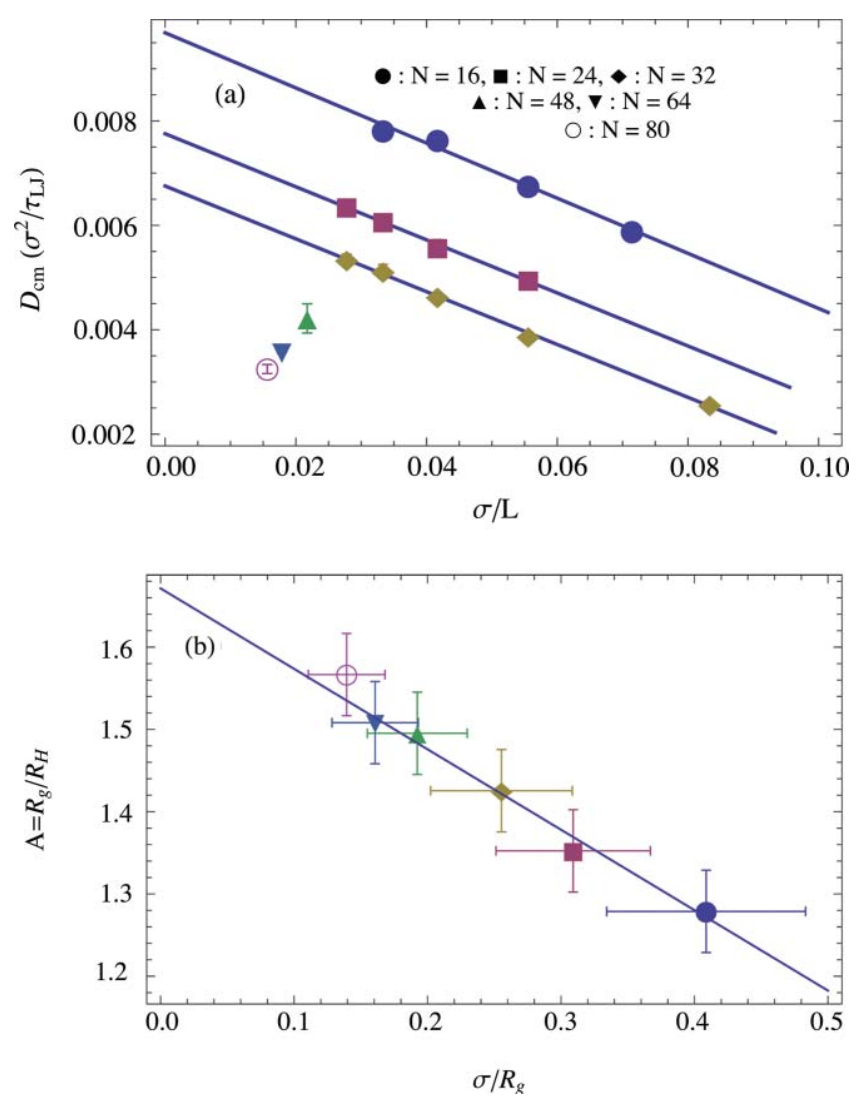

FIG. 11. (a) Diffusion coefficients of three short polymers scale linearly as a function of $1 / L$. We also show results for four longer chains at different $L$. The three asymptotic values at $L \rightarrow \infty$ are used in (b) together with the data for $(N, L)=(48,46)$ and $(64,56)$ to extract the ratio $A=R_{g} / R_{H}$ whose asymptotic value $1.67 \pm 0.05$ agrees within error bounds with the value $1.63 \pm 0.01$ from Ref. 70 for $\left\langle R_{g} / R_{I}\right\rangle$ in the excluded-volume case.

Results from similar SRD simulations ${ }^{64}$ suggest the existence of an additional term in Eq. (A1) of the form $D_{0}$ $=k_{\mathrm{B}} T /(\xi N)$, where $\xi$ is a friction parameter. To see this term, Liu and Dünweg ${ }^{72}$ have shown that one is to work strictly within the assumptions of Kirkwood theory so that the model exhibits clear time scale separation and, consequently, $D_{0}$ is visible at short times, but not in the long-time limit. For the sake of completeness, we performed a regression analysis for the data in Fig. 11(a) and found the existence of a $D_{0}$-like term to be below any statistical significance. Also, we see neither a ballistic regime nor an intermediate time scale in our MD-LB simulations. The lack of a clearly separated intermediate time scale means there is no theoretical argument that we should observe $D_{0},{ }^{72}$ which is also the argument that Liu and Dünweg give for $D_{0}$ not being observed in MD simulations. ${ }^{72}$ We have compared the SRD fluid parameters in Ref. 64 (such as density and viscosity) and they can be mapped to values in a similar range to ours. Hence, we attribute the difference between our and SRD simulations to the no-slip boundary condition at the surface of our monomers, which we impose by construction in our method, while the SRD may be able to slip more readily.

Following the notation in Ref. 60, we define

$$
\begin{aligned}
g_{2}(t)= & \left\langle\left(\left(\mathbf{r}_{c}(t+s)-\mathbf{r}_{\mathrm{cm}}(t+s)\right)\right.\right. \\
& \left.-\left(\mathbf{r}_{c}(s)-\mathbf{r}_{\mathrm{cm}}(s)\right)^{2}\right\rangle_{s},
\end{aligned}
$$



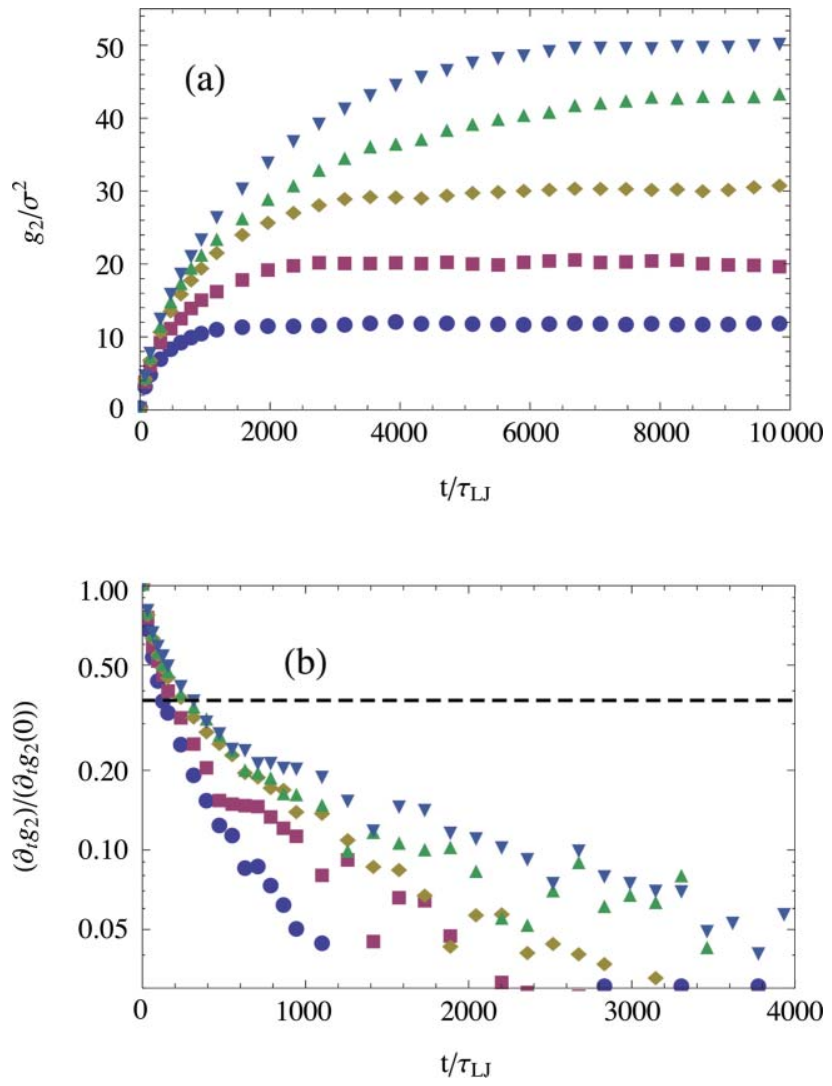

FIG. 12. (a) Mean squared displacement $g_{2}$ in center-of-mass frame, which flattens around the Zimm relaxation time $t_{Z}$. The data are for $N=32(\bullet), 48$ $(\square), 64(\checkmark), 80(\mathbf{\square})$ and $96(\mathbf{v})$. (b) The decay of the slope of $g_{2}$ is described as a two-stage exponential decay. A fit to the region below the horizontal $1 / e$ line gives an estimate of $t_{Z}=500, \ldots, 2000 \tau_{\mathrm{LJ}}$ for these chains.

for the mean squared displacement of central monomers in the CM frame of reference, where $\mathbf{r}_{c}(t)$, the average position of two monomers in the middle of the chain at time $t$ and $\mathbf{r}_{\mathrm{cm}}$, is the center-of-mass coordinate of the polymer. The Zimm time $t_{Z}$ is the time scale at which a polymer moves a distance comparable to its own radius, $t_{Z} \sim R_{g}^{2} / D_{\mathrm{cm}}$. We may estimate $t_{Z}$ from $g_{2}(t)$ based on the fact that after a time of order $t_{Z}$, the central monomers move along with the CM for which reason $g_{2}(t)$ should plateau for $t>t_{Z}$. We observe this transition in Fig. 12(a) to be very broad and estimate an upper bound for $t_{Z}$ to be in the range $1000-7000 \tau_{\mathrm{LJ}}$, depending on chain length. The smooth transition of Fig. 12(a) was also observed in Ref. 30. To make this more quantitative, the normalized slope of $g_{2}$ is plotted in log-linear scale in Fig. 12(b). Fitting straight lines to data in (b) below the $1 / e$ line indicated gives estimates $t_{Z}=500-2000 \tau_{\mathrm{LJ}}$ (as this is a "decay" time it is less than the above time estimates for when the plateau values were reached). Another estimate for the Zimm time is the translational time, $t_{\mathrm{tr}}=\left\langle R_{g}^{2}\right\rangle /\left(6 D_{\mathrm{cm}}\right)$, that is in the range between $500 \tau_{\mathrm{LJ}}(N=32)$ and $3200 \tau_{\mathrm{LJ}}(N=96)$. These estimates for $t_{Z}$ will be needed in Subsection IV E 3 as an upper bound for the dynamic scaling region.

\section{Dynamical scaling}

Brownian motion of a polymer is studied experimentally by means of dynamic light scattering. The time correlation of the intensity of the scattered light is embodied by the dynamic structure factor $S(\mathbf{k}, t)$, which is the time-dependent generalization of Eq. (48),

$$
\begin{aligned}
S(\mathbf{k}, t) & \equiv \frac{1}{N}\left\langle\hat{\rho}(\mathbf{k}, t+s) \hat{\rho}^{*}(\mathbf{k}, s)\right\rangle_{s} \\
& =\frac{1}{N} \sum_{m, n=1}^{N}\left\langle e^{i \mathbf{k} \cdot\left(\mathbf{r}_{m}(t+s)-\mathbf{r}_{n}(s)\right)}\right\rangle_{s},
\end{aligned}
$$

where $\mathbf{r}_{m}(t)$ is the position of the $m$ th monomer at time $t$ and \langle\rangle$_{s}$ denotes averaging over time.

We also define a dynamic structure factor in the centerof-mass coordinate system as

$$
S_{\mathrm{cm}}(\mathbf{k}, t) \equiv \frac{1}{N} \sum_{m, n=1}^{N}\left\langle e^{i \mathbf{k} \cdot\left(\tilde{\mathbf{r}}_{m}(t+s)-\tilde{\mathbf{r}}_{n}(s)\right)}\right\rangle_{s},
$$

where $\tilde{\mathbf{r}}_{m}(t)=\mathbf{r}_{m}(t)-\mathbf{r}_{\mathrm{cm}}(t)$. Here, $S_{\mathrm{cm}}(\mathbf{k}, t)$ is expected to be sensitive to intramolecular dynamics as CM motion is removed, whereas any short distances between the monomers observed by $S(\mathbf{k}, t)$ will be mostly along the backbone of the chain.

Within the assumptions of the Zimm model, the dynamic structure factor is expected to exhibit scaling in the form of

$$
S(k, t)=S(k, 0) F\left(k^{z} t\right)
$$

in the limit of an infinite dilute system and a long polymer chain for intermediate length scales, $2 \pi / R_{g}<k<2 \pi / l_{p}\left(l_{p}\right.$ is a microscopic cutoff), and times between the ballistic and the Zimm time in the system. ${ }^{61} F$ is a scaling function and $z$ is the dynamic scaling exponent.

The dynamic scaling exponent can be extracted based on Eq. (A1) by finding the value of $z$ that produces the best collapse of $S(k, t) / S(k, 0)$, or $S_{\mathrm{cm}}(k, t) / S_{\mathrm{cm}}(k, 0)$, for different $k$ as a function of $(k \sigma)^{z}\left(t / \tau_{\mathrm{LJ}}\right)$ in log-linear representation. We find the scaling exponent $z$ to match well with the prediction of the Zimm model, for which $z=3$, in the case of $S_{\mathrm{cm}}(\mathbf{k}, t)$. This is shown in Fig. 13(b) as a scaling collapse, which is seen to be sensitive for both the upper and the lower $k$ bound. The collapse is plotted as a function of $\left(k^{z} t\right)^{2 / 3}$ as it has been shown theoretically that $\log F(x) \sim x^{2 / 3}$ for $k^{3} k_{\mathrm{B}} T t /(6 \pi \eta) \gg 1 .^{73}$

Mussawisade et al. ${ }^{64}$ have confirmed by simulation a theoretic argument ${ }^{73}$ stating that the dynamic scaling exponent should, in fact, assume the value of $8 / 3$ similar to a semiflexible polymer in the laboratory frame for $k R_{g} \gg 1$. Figure 13(a) suggests that $z=2.43$ is quite optimal for a 32 -bead chain in the range $k \in[1.0,2.4] \sigma^{-1}$. Corresponding data for the 64-bead chain collapse best for $z=2.54 \pm 0.02$ in the laboratory frame of reference thus approaching the $8 / 3$ found by Winkler et al. ${ }^{73}$ We note that the scaling does not break down in Fig. 13(a) for length scales longer than $R_{g}$ such as $2 \pi /\left(1.0 \sigma^{-1}\right) \approx 6.3 \mathrm{~nm}$ in the laboratory frame even though $R_{g}=3.92 \mathrm{~nm}$. This is probably because most beads are within $R_{g}$ of the center of mass but separations between beads of $2 R_{g}$ are common and is the relevant length scale in the laboratory frame.

The upper $k$-bound of $2.4 \sigma^{-1}$ in Figs. 13(a) and 13(b) corresponds to a distance of $2.6 \sigma$ at which the normalized 

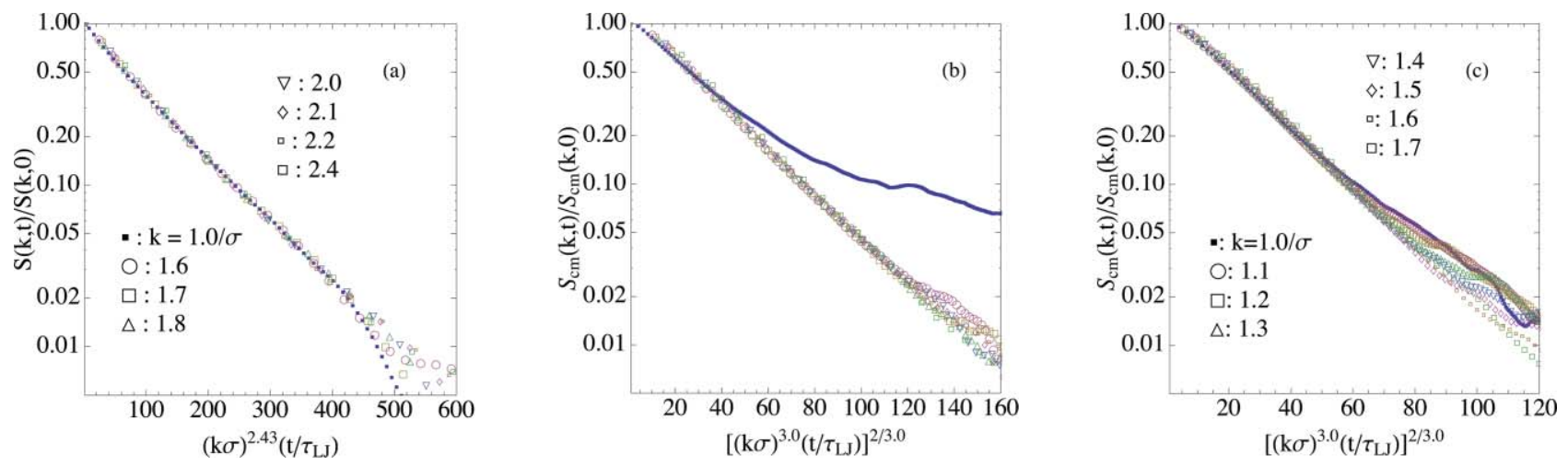

FIG. 13. The dynamic structure factor for a 32-bead polymer calculated (a) in the laboratory frame of reference and (b) in the center-of-mass frame of reference. Figures (a) and (b) show collapses of eight curves in the range $k \in[1.0,2.4] \sigma^{-1}$ and in (b) $k=1.0 \sigma^{-1}$ fails to collapse as it exceeds the maximal length scale present on the average in that frame. The collapse for $N=64$ in (c) in the center-of-mass frame is quite good, but the data still suffer from insufficient statistics for $(k \sigma)^{2}\left(t / \tau_{\mathrm{LJ}}\right)^{2 / 3}>60$.

bond-bond correlation function of Fig. 9 drops roughly to $1 / 3$. Larger $k$ values fail to collapse as well. The data in (a) and (b) collapses well up to $t=400 \tau_{\mathrm{LJ}}$ and $270 \tau_{\mathrm{LJ}}$ consistently with the estimates of $t_{Z}$ for $N=32$ in Sec. IV E 2.

Longer chains, such as the 64-bead polymer in Fig. 13(c), have longer relaxation times and it becomes increasingly difficult to obtain sufficient statistical averaging. Nevertheless, we observe the 64-bead chain to collapse in the center-ofmass reference frame over a decade, but for a smaller range of $k$ values. To understand this, we computed also the offdiagonal terms, $C_{p, q}(t) \equiv\left\langle\mathbf{X}_{p}(t+s) \cdot \mathbf{X}_{q}(s)\right\rangle_{s}, p \neq q$, of the Rouse matrix and found persistent correlations for $(p, q)$ $=(1,2), \ldots,(1,5)$ at a level of $C_{1, q}(t) / C_{1,1}(0) \approx 0.1$ even at $t=2000 \tau_{\mathrm{LJ}}$, but for $N=32$ the same normalized matrix elements were of the order of $\mathcal{O}\left(10^{-2}\right), \ldots, \mathcal{O}\left(10^{-3}\right)$ already at $t=100 \tau_{\mathrm{LJ}}$.

\section{SUMMARY AND CONCLUSIONS}

In this work, we have formulated the fluctuating LB equation using the well-known theory of linear stochastic differential equations and presented the connection between the variance of the underlying SDE and how it ties to the fluctuation-dissipation relation for the fluctuating stress tensor. We have presented how thermal fluctuations are implemented in an isothermal LB model in a way that maintains the desired local temperature stemming from fluctuations in the stress tensor, and how the ghost modes may be tuned to adjust the temperature, $T\left(L_{s}\right)$, as a function of subsystem size $L_{s}$. We have pointed out explicitly, in the context of the D3Q15 model, how hydrodynamic modes are coupled to lattice-specific modes and what is required for consistent thermalization. Also, we have applied a center-of-mass thermostat to our system by which it is possible to attain the same temperature at all length scales while momentum is still exactly conserved in any interaction between the solute and the solvent. This scheme also generalizes the model to a broader range of the speed of sound, which should allow straightforward generalization to nonideal fluids. Moreover, when MD particles such as colloids or polymer chains are coupled properly to the LB fluid, there is no longer any need to add ex- ternal Langevin noise to the solute. This leads to quantitative predictions of static and dynamical quantities that depend on the hydrodynamic coupling. Our method produces results for the center-of-mass diffusion coefficient of polymers whose finite-size effects are well approximated by theoretic calculations in the Oseen limit. ${ }^{60}$

\section{ACKNOWLEDGMENTS}

This work was supported by the Academy of Finland through its TransPoly and COMP CoE grants, by Tekes through its NanoTermo grant, by the Natural Science and Engineering Council of Canada, and by SharcNet. We also wish to thank CSC, the Finnish IT center for science, for allocation of computer resources. S.T.T.O. would like to thank the Magnus Ehrnrooth and the Alfred Kordelin Foundation for funding.

\section{APPENDIX A: DETAILS FOR THE D3Q15 LATTICE}

For a 15-velocity D3Q15 model in three dimensions, the mass density $\rho(\mathbf{r}, t)$ is $M^{0}(\mathbf{r}, t)$, where $m_{i}^{0}=1 \forall i$; the momentum densities $\rho u_{x}, \rho u_{y}, \rho u_{z}$ are $M^{1}, M^{2}$, and $M^{3}$, where $m_{i}^{1}=e_{i x}, m_{i}^{2}=e_{i y}, m_{i}^{3}=e_{i z} ; M^{4}$ through $M^{9}$ are associated with the six independent stress densities (in three dimensions), where $m_{i}^{a}$ with $a$ ranging from 4 to 9 are the upper triangular components of the tensor $e_{i \alpha} e_{i \beta}-\left(v_{c}^{2} / 3\right) \delta_{\alpha \beta}$, where $v_{c}$ is the lattice velocity $\Delta x / \Delta t ; M^{10}$ through $M^{13}$ are referred to as "ghost" densities and are associated with the third velocity moments of the distribution, where $m_{i}^{a}$, with $a$ from 10 to 13, are the four independent components (which we take to be the $x x y, x x z, x y y$, and $x y z$ components) of $e_{i \alpha} e_{i \beta} e_{i \gamma}-\left(v_{c}^{2} / 3\right)\left(e_{i \alpha} \delta_{\beta \gamma}+e_{i \beta} \delta_{\alpha \gamma}+e_{i \gamma} \delta_{\alpha \beta}\right)$; and finally $M^{14}$ is a ghost density associated with the (xxyy) one independent fourth moment of the distribution, with $m_{0}^{14}=\sqrt{2}$, $m_{1}^{14}$ through $m_{6}^{14}=-1 / \sqrt{2}$, and $m_{7}^{14}$ through $m_{14}^{14}=\sqrt{2}$. These vectors are written out in full in Table II.

The weights of Eq. (10) for the D3Q15 are $w_{0}=2 / 9$, $w_{1}$ through $w_{6}$ are $1 / 9$, and the rest are $1 / 72$. Similarly, the 
TABLE II. The eigenvectors $m^{a}$ of the moments $M^{a}$ in the D3Q15 model are presented as row vectors of the transformation matrix. $m^{12}, m^{10}$, and $m^{11}$ correspond to ghost momentum densities $M^{12}=J_{x}, M^{10}=J_{y}$, and $M^{11}=J_{z}$, respectively. The mixed diagonal vector $e_{i z} e_{i x} e_{i y}$ gives rise to mode $M^{13}=Q$.

\begin{tabular}{|c|c|c|c|c|c|c|c|c|c|c|c|c|c|c|c|c|}
\hline$\left[m^{0}\right]$ & & 1 & 1 & 1 & 1 & 1 & 1 & 1 & 1 & 1 & 1 & 1 & 1 & 1 & 1 & 1 \\
\hline$m^{1}$ & & 0 & $v_{c}$ & 0 & $-v_{c}$ & 0 & 0 & 0 & $v_{c}$ & $-v_{c}$ & $-v_{c}$ & $v_{c}$ & $v_{c}$ & $-v_{c}$ & $-v_{c}$ & $v_{c}$ \\
\hline$m^{2}$ & & 0 & 0 & $v_{c}$ & 0 & $-v_{c}$ & 0 & 0 & $v_{c}$ & $v_{c}$ & $-v_{c}$ & $-v_{c}$ & $v_{c}$ & $v_{c}$ & $-v_{c}$ & $-v_{c}$ \\
\hline$m^{3}$ & & 0 & 0 & 0 & 0 & 0 & $v_{c}$ & $-v_{c}$ & $v_{c}$ & $v_{c}$ & $v_{c}$ & $v_{c}$ & $-v_{c}$ & $-v_{c}$ & $-v_{c}$ & $-v_{c}$ \\
\hline$m^{4}$ & & $-\frac{v_{c}^{2}}{3}$ & $\frac{2 v_{c}^{2}}{3}$ & $-\frac{v_{c}^{2}}{3}$ & $\frac{2 v_{c}^{2}}{3}$ & $-\frac{v_{c}^{2}}{3}$ & $-\frac{v_{c}^{2}}{3}$ & $-\frac{v_{c}^{2}}{3}$ & $\frac{2 v_{c}^{2}}{3}$ & $\frac{2 v_{c}^{2}}{3}$ & $\frac{2 v_{c}^{2}}{3}$ & $\frac{2 v_{c}^{2}}{3}$ & $\frac{2 v_{c}^{2}}{3}$ & $\frac{2 v_{c}^{2}}{3}$ & $\frac{2 v_{c}^{2}}{3}$ & $\frac{2 v_{c}^{2}}{3}$ \\
\hline$m^{5}$ & & $-\frac{v_{c}^{2}}{3}$ & $-\frac{v_{c}^{2}}{3}$ & $\frac{2 v_{c}^{2}}{3}$ & $-\frac{v_{c}^{2}}{3}$ & $\frac{2 v_{c}^{2}}{3}$ & $-\frac{v_{c}^{2}}{3}$ & $-\frac{v_{c}^{2}}{3}$ & $\frac{2 v_{c}^{2}}{3}$ & $\frac{2 v_{c}^{2}}{3}$ & $\frac{2 v_{c}^{2}}{3}$ & $\frac{2 v_{c}^{2}}{3}$ & $\frac{2 v_{c}^{2}}{3}$ & $\frac{2 v_{c}^{2}}{3}$ & $\frac{2 v_{c}^{2}}{3}$ & $\frac{2 v_{c}^{2}}{3}$ \\
\hline$m^{6}$ & & $-\frac{v_{c}^{2}}{3}$ & $-\frac{v_{c}^{2}}{3}$ & $-\frac{v_{c}^{2}}{3}$ & $-\frac{v_{c}^{2}}{3}$ & $-\frac{v_{c}^{2}}{3}$ & $\frac{2 v_{c}^{2}}{3}$ & $\frac{2 v_{c}^{2}}{3}$ & $\frac{2 v_{c}^{2}}{3}$ & $\frac{2 v_{c}^{2}}{3}$ & $\frac{2 v_{c}^{2}}{3}$ & $\frac{2 v_{c}^{2}}{3}$ & $\frac{2 v_{c}^{2}}{3}$ & $\frac{2 v_{c}^{2}}{3}$ & $\frac{2 v_{c}^{2}}{3}$ & $\frac{2 v_{c}^{2}}{3}$ \\
\hline$m^{7}$ & $=$ & 0 & 0 & 0 & 0 & 0 & 0 & 0 & $v_{c}^{2}$ & $-v_{c}^{2}$ & $v_{c}^{2}$ & $-v_{c}^{2}$ & $v_{c}^{2}$ & $-v_{c}^{2}$ & $v_{c}^{2}$ & $-v_{c}^{2}$ \\
\hline$m^{8}$ & & 0 & 0 & 0 & 0 & 0 & 0 & 0 & $v_{c}^{2}$ & $v_{c}^{2}$ & $-v_{c}^{2}$ & $-v_{c}^{2}$ & $-v_{c}^{2}$ & $-v_{c}^{2}$ & $v_{c}^{2}$ & $v_{c}^{2}$ \\
\hline$m^{9}$ & & 0 & 0 & 0 & 0 & 0 & 0 & 0 & $v_{c}^{2}$ & $-v_{c}^{2}$ & $-v_{c}^{2}$ & $v_{c}^{2}$ & $-v_{c}^{2}$ & $v_{c}^{2}$ & $v_{c}^{2}$ & $-v_{c}^{2}$ \\
\hline$m^{10}$ & & 0 & 0 & $-\frac{v_{c}^{3}}{3}$ & 0 & $\frac{v_{c}^{3}}{3}$ & 0 & 0 & $\frac{2 v_{c}^{3}}{3}$ & $\frac{2 v_{c}^{3}}{3}$ & $-\frac{2 v_{c}^{3}}{3}$ & $-\frac{2 v_{c}^{3}}{3}$ & $\frac{2 v_{c}^{3}}{3}$ & $\frac{2 v_{c}^{3}}{3}$ & $-\frac{2 v_{c}^{3}}{3}$ & $-\frac{2 v_{c}^{3}}{3}$ \\
\hline$m^{11}$ & & 0 & 0 & 0 & 0 & 0 & $-\frac{v_{c}^{3}}{3}$ & $\frac{v_{c}^{3}}{3}$ & $\frac{2 v_{c}^{3}}{3}$ & $\frac{2 v_{c}^{3}}{3}$ & $\frac{2 v_{c}^{3}}{3}$ & $\frac{2 v_{c}^{3}}{3}$ & $-\frac{2 v_{c}^{3}}{3}$ & $-\frac{2 v_{c}^{3}}{3}$ & $-\frac{2 v_{c}^{3}}{3}$ & $-\frac{2 v_{c}^{3}}{3}$ \\
\hline$m^{12}$ & & 0 & $-\frac{v_{c}^{3}}{3}$ & 0 & $\frac{v_{c}^{3}}{3}$ & 0 & 0 & 0 & $\frac{2 v_{c}^{3}}{3}$ & $-\frac{2 v_{c}^{3}}{3}$ & $-\frac{2 v_{c}^{3}}{3}$ & $\frac{2 v_{c}^{3}}{3}$ & $\frac{2 v_{c}^{3}}{3}$ & $-\frac{2 v_{c}^{3}}{3}$ & $-\frac{2 v_{c}^{3}}{3}$ & $\frac{2 v_{c}^{3}}{3}$ \\
\hline$m^{13}$ & & 0 & 0 & 0 & 0 & 0 & 0 & 0 & $v_{c}^{3}$ & $-v_{c}^{3}$ & $v_{c}^{3}$ & $-v_{c}^{3}$ & $-v_{c}^{3}$ & $v_{c}^{3}$ & $-v_{c}^{3}$ & $v_{c}^{3}$ \\
\hline$m^{14}$ & & $\sqrt{2}$ & $-\frac{1}{\sqrt{2}}$ & $-\frac{1}{\sqrt{2}}$ & $-\frac{1}{\sqrt{2}}$ & $-\frac{1}{\sqrt{2}}$ & $-\frac{1}{\sqrt{2}}$ & $-\frac{1}{\sqrt{2}}$ & $\sqrt{2}$ & $\sqrt{2}$ & $\sqrt{2}$ & $\sqrt{2}$ & $\sqrt{2}$ & $\sqrt{2}$ & $\sqrt{2}$ & $\sqrt{2}$ \\
\hline
\end{tabular}

values of the normalization constants $N^{a}$ are

$$
\begin{aligned}
N^{a}= & (\underbrace{1,3 / v_{c}^{2}, 3 / v_{c}^{2}, 3 / v_{c}^{2}}_{\text {conserved densities }}, \\
& \underbrace{9 / 2 v_{c}^{4}, 9 / 2 v_{c}^{4}, 9 / 2 v_{c}^{4}, 9 / v_{c}^{4}, 9 / v_{c}^{4}, 9 / v_{c}^{4}}_{\text {stress densities }}, \\
& \underbrace{\left.27 / 2 v_{c}^{6}, 27 / 2 v_{c}^{6}, 27 / 2 v_{c}^{6}, 9 / v_{c}^{6}, 1\right)}_{\text {ghost densities }} .
\end{aligned}
$$

Due to Eq. (A1), the external forcing terms $p_{i}$ can be expressed analogously to Eq. (10) using the conversion matrix $m_{i}^{a}$ of the D3Q15 lattice (see Table II) as

$$
p_{i}=w_{i}\left(\frac{3 F_{\alpha}}{v_{c}^{2}} e_{i \alpha}+\frac{9\left(u_{\alpha} F_{\beta}+F_{\alpha} u_{\beta}\right)}{2 v_{c}^{4}} H_{\alpha \beta}\right),
$$

where $H_{\alpha \beta}=e_{i \alpha} e_{i \beta}-\left(v_{c}^{2} / 3\right) \delta_{\alpha \beta}$.

Equation (13) also yield expressions for the local relaxation of the second moment $[a=5, \ldots, 9$ in Eq. (13) $]$ as

$$
\begin{gathered}
\partial_{t} \Pi_{\alpha \beta}+\partial_{\gamma}\left[\frac{1}{3} \rho u_{\gamma} v_{c}^{2}+J_{\gamma}+\delta_{\gamma \alpha}\left(\frac{2}{3} \rho u_{\beta} v_{c}^{2}-J_{\beta}\right)\right] \\
=-\frac{1}{\tau}\left(\Pi_{\alpha \beta}-\Pi_{\alpha \beta}^{\mathrm{eq}}\right), \quad \text { if } \alpha=\beta, \\
\partial_{t} \Pi_{\alpha \beta}+\partial_{\alpha}\left[\frac{1}{3} \rho u_{\beta} v_{c}^{2}+J_{\beta}\right]+\partial_{\beta}\left[\frac{1}{3} \rho u_{\alpha} v_{c}^{2}+J_{\alpha}\right] \\
+\left(1-\delta_{\gamma \alpha}-\delta_{\gamma \beta}\right) \partial_{\gamma} Q=-\frac{1}{\tau}\left(\Pi_{\alpha \beta}-\Pi_{\alpha \beta}^{\mathrm{eq}}\right), \quad \text { if } \quad \alpha \neq \beta,
\end{gathered}
$$

where $J_{x}=M^{12}, J_{y}=M^{10}$, and $J_{z}=M^{11}$ are ghost momentum densities corresponding to $m^{12}, m^{10}$, and $m^{11}$, respectively. Their name comes from the fact that they have exactly the same nonzero components as their counterparts $m^{1}, m^{2}$, and $m^{3} \cdot Q=M^{13}$ is the third-order moment $\sum_{i} f_{i} e_{i x} e_{i y} e_{i z}$. This is made evident by Table II that lists all basis vectors $m^{a}$.
Equation (A4) reveals how the ghost momenta $J_{\alpha}$ function as source terms for the second moment since they are inside the divergence term. Equation (13) also reveal how the physical second moments act as source terms for the ghost momenta $J_{\alpha}[a=10,11,12$ in Eq. (13), but we write out only the $x$ component],

$$
\begin{gathered}
\partial_{t} J_{x}+\frac{1}{3} \partial_{x}\left[\Pi_{y y}+\Pi_{z z}-2 \rho+\frac{\sqrt{2} K}{3}\right] \\
+\frac{2}{3}\left(\partial_{y} \Pi_{x y}+\partial_{z} \Pi_{x z}\right)=-\frac{J_{x}-J_{x}^{\mathrm{eq}}}{\tau},
\end{gathered}
$$

where $K$ denotes the 14 th density $M^{14}$. Together these equations suggest that if the velocity field of the fluid is to exhibit equipartition, the $J \mathrm{~S}$ must not interfere with leading order viscous stress ${ }^{39}$ nor drain fluctuations from the second moments as the $J$ s are not present in the momentum equation [see Eqs. (17) and (16b)]. This fact imposes $J_{\alpha}^{\mathrm{eq}}=0$ based on Eq. (A4) and a lengthy analysis shows that $Q^{\text {eq }}$ may be chosen to be $\rho u_{x} u_{y} u_{z}$.

At this point, by adding the dynamic equations for $J_{y}$ and $J_{z}$ to Eq. (A6) and by leaving only first-order terms of the derivative expansion and the leading order stress $\Pi_{\alpha \beta}^{0}$ $=P_{\alpha \beta}+\rho u_{\alpha} u_{\beta}$, we arrive at Eq. (18) in the text.

\section{APPENDIX B: ON THE IMPLEMENTATION OF THE ALGORITHM}

We describe here the implementation of the present algorithm. The program is organized as follows.

- Scale parameters to have units $\Delta x, \Delta t$, and $m_{\mathrm{LJ}}$.

- Define lattice structure, i.e., vectors $\mathbf{e}_{i}$, precompute static transformation matrix $m_{i}^{a}$ (see our choice for D3Q15 above in Appendix A) and normalization factors $N^{a}$ [Eqs. (12) and (A1)]. Initialize fluid by setting $\rho(\mathbf{x}, 0)=$ $\rho_{0}, u_{\alpha}(\mathbf{x}, 0)=0.0$ and $f_{i}(\mathbf{x}, 0)=w_{i} \rho(\mathbf{x}, 0)$. The equilibria $h_{i}^{\mathrm{eq}}(\mathbf{x}, 0)$ are initialized using Eq. (10) from $\rho(\mathbf{x}, 0), u_{\alpha}(\mathbf{x}, 0)$ 
and $P_{\alpha \beta}(\mathbf{x}, 0)=v_{s}^{2} \rho(\mathbf{x}, 0) \delta_{\alpha \beta}$ together with $\zeta^{a}(\mathbf{x}, 0)$ as described in Step 3 of Sec. III B.

- Place (possibly microcanonically equilibrated) MD particles in fluid, set their CM velocity to zero.

- Run simulation:

loop $\mathrm{n}=1:(\mathrm{Neq}+\mathrm{Nmax})$

1. Compute $h_{i}^{\mathrm{eq}}(\mathbf{x}, n-1) \forall \mathbf{x}$ using Eq. (28), the relation $g_{i}^{\mathrm{eq}}=f_{i}^{\mathrm{eq}}+\tau p_{i}$ and via Eqs. (10) and (31).

2. Compute $f_{i}(\mathbf{x}, n) \forall \mathbf{x}$ using Eq. (27).

3. Compute $\rho(\mathbf{x}, n), u_{\alpha}(\mathbf{x}, n) \forall \mathbf{x}$ using Eq. (9).

4. Update MD part, compute frictional force.

5. Sample data.

- Output end-of-run data.

We note that calculation of $P_{\alpha \beta}(\mathbf{x}, n)$ must take into account corrections to reduce errors in non-Galilean invariant terms as presented in Ref. 43.

To set the center-of-mass velocity of the total system to a desired value, we first focus on the momentum-conservation equation with a constant shift $\mathbf{U}=\left(U_{x}, U_{y}, U_{z}\right)$ in velocity that does not affect the stress as it is a Galilean-invariant transformation and, as such, it does not result in velocity gradients,

$$
\begin{aligned}
& \partial_{t}\left(\rho\left(u_{\alpha}-U_{\alpha}\right)+\partial_{\beta}\left(\rho\left(u_{\alpha}-U_{\alpha}\right)\left(u_{\beta}-U_{\beta}\right)\right)=\partial_{\beta} \sigma_{\alpha \beta}\right. \\
& \Leftrightarrow \quad \partial_{t}\left(\rho u_{\alpha}\right)+\partial_{\beta}\left(\rho u_{\alpha} u_{\beta}\right) \\
& \quad-\partial_{t}\left(\rho U_{\alpha}\right)-\partial_{\beta}\left(\rho u_{\alpha} U_{\beta}+\rho u_{\beta} U_{\alpha}-\rho U_{\alpha} U_{\beta}\right) \\
& =\partial_{\beta} \sigma_{\alpha \beta} .
\end{aligned}
$$

The constant shift is realized by generating populations $f_{i}^{(\mathrm{CM})}(\mathbf{x}, t)$ that are subtracted from the $f_{i}(\mathbf{x}, t)$. The terms containing $U_{\alpha}$ tell us what the first $\left(M_{(\mathrm{CM})}^{1}\right.$ through $\left.M_{(\mathrm{CM})}^{3}\right)$ and second $\left(M_{(\mathrm{CM})}^{4}\right.$ through $\left.M_{(\mathrm{CM})}^{9}\right)$ moments of $f_{i}^{(\mathrm{CM})}(\mathbf{x}, t)$ should be for the correct shift to be realized. The moments are listed in Table III. The nonzero ghost moment $M_{(\mathrm{CM})}^{13}$ is

TABLE III. Moments $M_{(\mathrm{CM})}^{a}$ that are used to construct the populations $f_{i}^{(\mathrm{CM})}$ using Eq. (10) and to set the center-of-mass velocity to a desired value.

\begin{tabular}{lc}
\hline \hline$a$ & $M_{(\mathrm{CM})}^{a}$ \\
\hline 0 & 0 \\
1 & $\rho U_{x}$ \\
2 & $\rho U_{y}$ \\
3 & $\rho U_{z}$ \\
4 & $\rho\left(2 u_{x} U_{x}-U_{x}^{2}\right)$ \\
5 & $\rho\left(2 u_{y} U_{y}-U_{y}^{2}\right)$ \\
6 & $\rho\left(2 u_{z} U_{z}-U_{z}^{2}\right)$ \\
7 & $\rho\left(u_{x} U_{y}+u_{y} U_{x}-U_{x} U_{y}\right)$ \\
8 & $\rho\left(u_{y} U_{z}+u_{z} U_{y}-U_{y} U_{z}\right)$ \\
9 & $\rho\left(u_{x} U_{z}+u_{z} U_{x}-U_{x} U_{z}\right)$ \\
10 & 0 \\
11 & 0 \\
12 & 0 \\
13 & $\rho\left(u_{x} u_{y} U_{z}+u_{x} U_{y} u_{z}+U_{x} u_{y} u_{z}-u_{x} U_{y} U_{z}-U_{x} u_{y} U_{z}-\right.$ \\
14 & $\left.U_{x} U_{y} u_{z}+U_{x} U_{y} U_{z}\right)$ \\
\hline \hline
\end{tabular}

constructed analogously noting that $Q^{\mathrm{eq}}=M_{\mathrm{eq}}^{13}=\rho u_{x} u_{y} u_{z}$. Figure 1 is the result of setting $U_{\alpha}(t)=V_{\alpha}(t)-U_{\alpha}^{(\mathrm{R})}(t)$, where $V_{\alpha}$ is the center-of-mass velocity of Eq. (38) and $U_{\alpha}^{(\mathrm{R})}$ is the random velocity due to the heat bath that is propagated in time using the Euler-Maruyama approximation ${ }^{46}$ as

$$
\begin{aligned}
U_{\alpha}^{(\mathrm{R})}(t+\Delta t)= & (1-\chi \Delta t) U_{\alpha}^{(\mathrm{R})}(t) \\
& +\sqrt{2 k_{\mathrm{B}} T \chi / M_{T}} \sqrt{\Delta t} \mathcal{N}(0,1) ; \\
U_{\alpha}^{(\mathrm{R})}(0)= & 0,
\end{aligned}
$$

where $\mathcal{N}(0,1)$ is a normally distributed random number of zero mean and unit variance.

The choice of $\chi$ in Eq. (38) depends on the size of and the speed of sound in the system. We found that $\chi=700 \mathrm{~ns}^{-1}$ worked for the systems tested $(L=30$ and $50 \mathrm{~nm})$. The value of $\chi$ corresponds to a time scale $\chi^{-1}$, which should be larger than the simulation time step $\Delta t$ so that the relaxation is resolved by the explicit solver of Eq. (B2). Also, the solver does not overshoot for $\chi \Delta t<1$.

${ }^{1}$ A. Einstein, Ann. Phys. (Leipzig) 17, 549 (1905).

${ }^{2}$ E. Falck, J. M. Lahtinen, I. Vattulainen, and T. Ala-Nissila, Eur. Phys. J. E 13, 267 (2004)

${ }^{3}$ S. Chen and G. D. Doolen, Ann. Rev. Fluid Mech. 30, 329 (1998).

${ }^{4}$ M. Karttunen et al. (eds), Novel Methods in Soft Matter Simulations, Lecture Notes on Physics Vol. 640 (Springer-Verlag, Berlin, 2004).

${ }^{5}$ D. M. Anderson, G. B. McFadden, and A. A. Wheeler, Ann. Rev. Fluid Mech. 30, 139 (1998).

${ }^{6}$ M. R. Swift, W. R. Osborn, and J. M. Yeomans, Phys. Rev. Lett. 75, 830 (1995).

${ }^{7}$ C. Denniston, E. Orlandini, and J. M. Yeomans, Comp. Theor. Poly. Sci. 11, 389 (2001)

${ }^{8}$ P. D. Olmsted, O. Radulescu, and C.-Y. D. Lu, J. Rheol. 44, 256 (2000); C.-Y. D. Lu, P. D. Olmsted, and R. C. Ball, Phys. Rev. Lett. 84, 642 (2000).

${ }^{9}$ E. Kuusela and T. Ala-Nissila, Phys. Rev. E 63, 061505 (2001); E. Kuusela, J. M. Lahtinen, and T. Ala-Nissila, Phys. Rev. E 69, 066310 (2004).

${ }^{10}$ E. Kuusela, J. M. Lahtinen, and T. Ala-Nissila, Phys. Rev. Lett. 90, 094502 (2003).

${ }^{11}$ J. M. Yeomans, Annual Reviews of Computational Physics VII, edited by D. Stauffer (World Scientific, Singapore, 1999), p. 61.

${ }^{12} \mathrm{~S}$. A. Safran, Statistical Thermodynamics of Surfaces, Interfaces and Membranes (Westview, New York NY, 1994); D. G. A. L. Aarts, M. Schmidt, and H. N. W. Lekkerkerker, Science 304847 (2004).

${ }^{13} \mathrm{~F}$. Franks Water, A Matrix of Life, 2nd ed. (The Royal Society of Chemistry, Cambridge UK, 2000).

${ }^{14}$ J. T. Padding and A. A. Louis, Phys. Rev. E 77, 011402 (2008).

${ }^{15}$ Y. Lin, H. Skaff, T. Emrick, A. D. Dinsmore, and T. P. Russell, Science 299, 299 (2003); Y. Lin, A. Böker, H. Skaff, D. Cookson, A.D. Dinsmore, T. Emrick, and T. P. Russell, Langmuir 21, 191 (2005).

${ }^{16}$ M. Ripoll, K. Mussawisade, R. G. Winkler, and G. Gompper, Europhys. Lett. 68, 106 (2004).

${ }^{17}$ R. D. Groot and P. B. Warren, J. Chem. Phys. 107, 4423 (1997).

${ }^{18}$ C. P. Lowe, Europhys. Lett. 47, 145 (2004).

${ }^{19}$ E. A. J. F. Peters, Europhys. Lett. 66, 311 (2004).

${ }^{20}$ A. J. C. Ladd, J. Fluid Mech. 271, 285 (1994); ibid. 311 (1994).

${ }^{21}$ L. D. Landau and E. M. Lifschitz, Statistical Physics (Pergamon, New York, 1980).

${ }^{22}$ P. Español, Phys. A 248, 77 (1998).

${ }^{23}$ K. Huang, Statistical Mechanics, 2nd ed. (Wiley, New York, 1987).

${ }^{24}$ S. Succi, The Lattice Boltzmann Equation for Fluid Dynamics and Beyond (Oxford University Press, New York, 2001).

${ }^{25}$ R. Adhikari, K. Stratford, M. E. Cates, and A. J. Wagner, Europhys. Lett. 71, 473 (2005).

${ }^{26}$ B. Dünweg, U. D. Schiller, and A. J. C. Ladd, Phys. Rev. E 76, 036704 (2007).

${ }^{27}$ N.-Q. Nguyen and A. J. C. Ladd, Phys. Rev. E 66, 046708 (2002).

${ }^{28}$ M. E. Cates, K. Stratford, R. Adhikari, P. Stansell, J.-C. Desplat, I. Pagonabarraga, and A. J. Wagner, J. Phys.: Condens. Matter 16, 3903 (2004). 
${ }^{29}$ P. Ahlrichs and B. Dünweg, Int. J. Mod. Phys. C 9, 1429 (1998).

${ }^{30}$ P. Ahlrichs and B. Dünweg, J. Chem. Phys. 111, 8225 (1999).

${ }^{31}$ T. T. Pham, U. D. Schiller, J. R. Prakash, and B. Dünweg, J. Chem. Phys. 131, 164114 (2009).

${ }^{32}$ A. Chatterji and J. Horbach, J. Chem. Phys. 122, 184903 (2005).

${ }^{33}$ Y. Iwashita, Y. Nakayama, and R. Yamamoto, J. Phys. Soc. Jpn. 77, 074007 (2008).

${ }^{34}$ S. T. T. Ollila, C. J. Smith, and C. Denniston, "Hydrodynamic consistency of particles in the lattice-Boltzmann method," J. Chem. Phys. (to be published).

${ }^{35}$ L. E. Reichl, A Modern Course in Statistical Physics, 2nd ed. (Wiley, New York, 1998).

${ }^{36}$ M. Gross, R. Adhikari, M. E. Cates, and F. Varnik, Phys. Rev. E 82056714 (2010).

${ }^{37}$ A. Donev, E. Vanden-Eijnden, A. L. Garcia, and J. B. Bell, Communications in Applied Mathematics and Computational Science 5, 2 pp. 149-197 (2010).

${ }^{38}$ P. L. Bhatnagar, E. P. Gross, and M. Krook, Phys. Rev. 94, 511 (1954).

${ }^{39}$ P. J. Dellar, Phys. Rev. E 65, 036309 (2002).

${ }^{40}$ S. Ansumali and I. V. Karlin, Phys. Rev. Lett. 95, 260605 (2005); N. I. Prasianakis and I. V. Karlin, Phys. Rev. E 76, 016702 (2007).

${ }^{41}$ I. Pagonabarraga, Lattice Boltzmann Modeling of Complex Fluids: Colloidal Suspensions and Fluid Mixtures, Lecture Notes on Physics Vol. 640 (Springer-Verlag, Berlin Heidelberg, 2004), pp. 279-309.

${ }^{42}$ A. J. C. Ladd and R. Verberg, J. Stat. Phys. 104, 1191 (2001).

${ }^{43}$ D. J. Holdych, D. Rovas, J. G. Georrgiadis, and R. O. Buckius, Int. J. Mod. Phys. C 9, 1393 (1998).

${ }^{44}$ D. d'Humières, I. Ginzburg, M. Krafczyk, P. Lallemand, and L.-S. Luo, Philos. Trans. R. Soc. London, Ser. A 360, 437 (2002).

${ }^{45}$ B. Øksendal, Stochastic Differential Equations, 5th ed. (Springer-Verlag, Berlin, 1998).

${ }^{46}$ P. E. Kloeden and E. Platen, Numerical solution of Stochastic Differential Equations, 2nd ed. (Springer-Verlag, Berlin, 1995).

${ }^{47}$ J. C. Strikwerda, Finite Difference Schemes and Partial Differential Equations, 2nd ed. (SIAM, USA, 2004), p. 3.

${ }^{48}$ R. W. Nash, R. Adhikari, and M. E. Cates, Phys. Rev. E 77, 026709 (2008).

${ }^{49}$ S. Chikatamarla and I. V. Karlin, Phys. Rev. E 79, 046701 (2009).
${ }^{50}$ G. H. Golub and C. F. van Loan, Matrix Computations, 3rd ed. (The Johns Hopkins University Press, Baltimore, MD, 1996).

${ }^{51}$ S. M. Karim and L. Rosenhead, Rev. Mod. Phys. 24, 108 (1952).

${ }^{52}$ L. D. Landau and E. M. Lifschitz, Fluid Mechanics (Pergamon, New York, 1984).

${ }^{53}$ G. Stokes, Mathematical and Physical Papers Volume I located at www.archive.org/details/mathphyspapers01stokrich (Cambridge University Press, Cambridege, England, 1880).

${ }^{54}$ E. H. Hauge and A. Martin-Lof, J. Stat. Phys. 7, 259 (1973).

${ }^{55}$ R. Zwanzig and M. Bixon, J. Fluid Mech. 69, 21 (1975).

${ }^{56}$ T. V. Lokotosh and N. P. Malomuzh, Phys. A 286, 474 (2000).

${ }^{57}$ A. Donev, A. L. Garcia, and B. J. Alder, Phys. Rev. Lett. 101, 075902 (2008).

${ }^{58}$ D. Frenkel and B. Smit, Understanding Molecular Simulation (Academic, New York, 2002).

${ }^{59}$ A. Malevanets and J. M. Yeomans, Europhys. Lett. 52, 231 (2000).

${ }^{60}$ B. Dünweg and K. Kremer, J. Chem. Phys. 99, 6983 (1993).

${ }^{61}$ M. Doi and S. F. Edwards, The Theory of Polymer Dynamics, (Oxford University Press, London, 1986).

${ }^{62}$ G. S. Grest and K. Kremer, Phys. Rev. A 33, 3628 (1986).

${ }^{63}$ B. Li, N. Madras, and A. D. Sokal, J. Stat. Phys. 80, 661 (1995).

${ }^{64}$ K. Mussawisade, M. Ripoll, R. G. Winkler, and G. Gompper, J. Chem. Phys. 123, 144905 (2005).

${ }^{65}$ H.-P. Hsu, W. Paul, and K. Binder, Macromolecules 43, 3094 (2010).

${ }^{66}$ A. Kopf, B. Dünweg, and W. Paul, J. Chem. Phys. 107, 6945 (1997).

${ }^{67}$ J. M. Polson and J. P. Gallant, J. Chem. Phys. 124, 184905 (2006).

${ }^{68}$ O. Punkkinen, E. Falck, I. Vattulainen, and T. Ala-Nissila, J. Chem. Phys. 122, 094904 (2005).

${ }^{69}$ C. W. J. Beenakker, J. Chem. Phys. 85, 1581 (1986).

${ }^{70}$ B. Dünweg, D. Reith, M. Steinhauser, and K. Kremer, J. Chem. Phys. 117, 914 (2002).

${ }^{71}$ P. Sunthar and J. R. Prakash, Europhys. Lett. 75, 77 (2006).

${ }^{72}$ B. Liu and B. Dünweg, J. Chem. Phys. 118, 8061 (2003).

${ }^{73}$ R. G. Winkler, L. Harnau, and P. Reineker, Macromol. Theory Simul. 6, 1007 (1997). 